Pacific Journal of Mathematics

MESURES CYLINDRIQUES, MESURES VECTORIELLES ET
QUESTIONS DE CONCENTRATION CYLINDRIQUE 


\title{
MESURES CYLINDRIQUES, MESURES VECTORIELLES ET QUESTIONS DE CONCENTRATION CYLINDRIQUE
}

\author{
ANDRE GOLDMAN
}

\begin{abstract}
Let $E$ be a locally convex space and $\vec{m}: \Sigma \rightarrow E$ an $E$-valued vector measure, absolutely continuous with respect to a scalar measure $\mu$; to each pair $(\vec{m}, \mu)$ we can associate a cylindrical mesure $\lambda$ on $E$. It is shown some Radon-Nikodym theorems can be deduced from the properties of the cylindrical concentration of $\lambda$. It is shown also that the $\sigma$-dentability properties of certain subsets of $E$ are closely related to some particular conditions of cylindrical concentration (these conditions are introduced by $A$. Badrikian and $S$. Chevet in the recent book "Mesures cylindriques, espaces de Wiener et fonctions aléatoires gaussiennes"). Finally, we consider the particular case of a measure $\vec{m}$ which takes its values into the positive cone of a measure space such as $M_{t}(T)$ (tight measures), $M_{t}(T)$ (smooth measures), or $M^{\infty}(T)$ (separable measures introduced by Dudley).
\end{abstract}

La théorie des mesures vectorielles est étroitement liée à celle des mesures cylindriques. En effet si $\vec{m}: \Sigma \rightarrow E$ est une mesure vectorielle à valeurs dans un espace localement convexe (en abrégé elc) $E$ absolument continue par rapport à une probabilité $\mu \operatorname{sur}(\Omega, \Sigma)$ l'application $L$, qui à $x^{\prime} \in E^{\prime}$ (dual de $E$ ) associe la densité scalaire $f_{x^{\prime}} \in L^{1}(\Omega, \Sigma, \mu)$ de la mesure $x^{\prime} \circ \vec{m}$ par rapport à $\mu$, définit sur $E$ une mesure cylindrique $\lambda$. Il est bien classique que si $E$ est un espace de Banach et $\vec{m}$ à variation bornée alors le couple $(\vec{m}, \mu)$ admet une densité Bochner-intégrable si et seulement si $\lambda$ est de Radon sur $E$ (ou sur $\left(E, \sigma\left(E, E^{\prime}\right)\right)$ ). Cette correspondance entre mesure vectorielle et mesure cylindrique fut par exemple exploitée, dans le cadre des espaces de Banach, par L. Schwartz et B. Maurey [20] qui retrouvent de cette manière des résultats de Uhl, Rieffel, Stegall etc.

On se propose d'approfondir l'influence mutuelle des propriétés du couple $(\vec{m}, \mu)$ et de la mesure cylindrique $\lambda$ associée. A cet effet, on prendra du recul par rapport à la situation classique des espaces de Banach en se plaçant d'emblée dans le contexte des espaces localement convexes généraux.

Dans le premier paragraphe on précise le cadre de l'étude et on rappelle les relations connues qui existent entre le couple $(\vec{m}, \mu)$ et la mesure cylindrique $\lambda$ associée. On retrouve ainsi les théorèmes du type Radon-Nikodym, de Metivier [16], de Uhl [24] etc. Ces 
résultats sont bien connus; nous en donnerons des démonstrations très sommaires.

Dans le paragraphe suivant on donne, en faisant intervenir les propriétés des ensembles $S_{A}=\left\{\vec{m}(B) / \mu(B) ; B \in \Sigma_{A}, \mu(B) \neq 0\right\}$, un critère important de concentration cylindrique pour la mesure $\lambda$. On en déduit des conditions suffisantes pour que $\lambda$ soit de Radon $\operatorname{sur} E$; si de plus, pour tout $\varepsilon>0$, la mesure $\lambda$ est concentrée à $\varepsilon$-près sur des disques faiblement compacts de $E$ (pour la topologie $\sigma\left(E, E^{\prime}\right)$ ) alors il existe une densité faible $\vec{f}: T \rightarrow E$. Les résultats redonnent en particulier les théorèmes du type Radon-Nikodym, de J. Pellaumaill [17], J. Kupka [12] et D. J. Hebert Jr. [8]. Dans le cas particulier où $E$ est un espace quasi-complet souslinien, on obtient des conditions nécessaires et suffisantes pour qu'il existe une densité $\Sigma$-mesurable, ce qui permet de retrouver certains des critères établis par E. F. Thomas [22] pour qu'une fonction intégrable au sens de Pettis soit sommable.

Le troisième paragraphe traite de l'influence des propriétés de $\sigma$-dentabilité [18] des ensembles $S_{A}$ sur la mesure $\lambda$. A cet effet, nous ferons appel à de récents résultats de $\mathrm{A}$. Badrikian et $\mathrm{S}$. Chevet [3] concernant des conditions de concentration cylindrique. On dira qu'une probabilité cylindrique $\lambda$ sur $E$ vérifie la condition (B) lorsque pour tout $\varepsilon>0$ et tout voisinage de zéro $V$ dans $E$ il existe un compact $K_{V}^{\varepsilon}$ tel que l'on ait $\lambda^{*}\left(K_{V}^{\varepsilon}+V\right) \geqq 1-\varepsilon$. Si toute mesure cylindrique sur $E$ vérifiant (B) est de Radon alors on dit, en suivant [3], que $E$ est un "bon espace": par exemple, les espaces de Fréchet sont de "bons espaces." Le lien entre la $\sigma$-dentabilité et la condition (B) est très précis: si pour tout $A \in \Sigma, \mu(A) \neq 0$ il existe $B \in \Sigma_{A}$, $\mu(B) \neq 0$ tel que $S_{B}$ soit $\sigma$-dentable, alors $\lambda$ vérifie la condition (B). Ceci redonne dans le cas où $E$ est un espace de Banach (donc un "bon espace") le théorème classique de Rieffel [18]. En fait, l'étude des conditions équivalentes à (B) nous amène à introduire des notions de $\sigma$-dentabilité quelque peu plus faible que celle définie par Rieffel [18], et ceci même pour les espaces de Banach. Finalement on montre, en suivant de près le résultat similaire de Maynard [15], que tous les bornés d'un espace quasi-complet $E$ sont $\sigma$-dentables si et seulement si toute mesure $\lambda$ associée aux couples $(\vec{m}, \mu)$ vérifie la condition (B). Si cette condition est vérifiée, l'espace $E$ n'est pas pour autant un espace de Radon-Nikodym faible. Pour des exemples d'une telle situation on consultera le Paragraphe IV. Inversement, un couple $(\vec{m}, \mu)$ peut très bien admettre une densité faible $\overrightarrow{f:} T \rightarrow E$ sans que pour autant la mesure cylindrique associée $\lambda$ soit de Radon (et cela, même si $E$ est un espace de Banach).

Dans le dernier chapitre on envisage le cas particulier des couples 
$(\vec{m}, \mu)$ où la mesure vectorielle $\vec{m}$ est à valeurs dans le cône positif $M^{+}(\beta T)$ des mesures de Radon sur le compactifié de Stone-Čech d'un espace complètement régulier $T$. Les résultats essentiels sont les suivants. Si $\vec{m}$ est à valeurs dans l'espace $M_{t}^{+}(T)$ [1] des mesures de Radon sur $T$ alors il existe toujours une densité faible $\overrightarrow{f:} \Omega \rightarrow M_{t}^{+}(T)$; de plus, la mesure cylindrique $\lambda$ est de Radon sur $M_{t}^{+}(T)$ et, pour tout $\varepsilon>0$, concentrée à $\varepsilon$-près sur des ensembles $H_{\varepsilon}$ vérifiant la condition de Prokhorov. Dans le cas de l'espace $M_{\sigma}^{+}(T)$ des mesures de Baire sur $T$, si toute mesure provenant d'un couple $(\vec{m}, \mu)$ est de Radon alors le replété $\nu T$ [4] de $T$ est universellement Radonmesurable (ou hyporadonien en suivant [21]). Si on suppose que $T$ est métrisable séparable alors la condition $T$ est radonien équivaut au fait que tout couple $(\vec{m}, \mu)$ soit densitable. Ce dernier résultat permet de donner des exemples d'espaces complets dans lesquels toutes les parties bornées sont $\sigma$-dentables et qui ne sont pas des espaces de Radon-Nikodym faibles. Pour établir ce dernier point, on fera appel aux résultats obtenus par J. Berruyer et B. Ivol [4] ainsi que par M. Rome [19] dans l'étude des espaces de mesures $M_{\sigma}(T)$ et $M^{\infty}(T)$.

I. Etude preliminaire. Dans tout ce travail on désignera par $\vec{m}: \Sigma \rightarrow E$ une mesure vectorielle à valeurs dans $E$, définie sur une tribu $\Sigma$ construite sur un ensemble $T$. L'espace $E$ est localement convexe et supposé toujours séparé. On notera encore par $\mu$ une mesure scalaire positive définie sur l'espace mesurable $(T, \Sigma)$. Une fonction $\vec{f}: T \rightarrow E$ est dite totalement mesurable lorsqu'elle est $\Sigma$ mesurable (relativement à la tribu borélienne de $E$ ) et d'image presque séparable sur chaque ensemble $\mu$-intégrable $A \in \Sigma$. En fait cette notion n'a d'intérêt que si $E$ est métrisable. Ainsi, à l'exception de ce cas, on distinguera essentiellement les différentes notions de fonctions mesurables:

(a) fonctions scalairement mesurables

(b) fonctions $\Sigma$-mesurables et

(c) fonctions Lusin-mesurables, lorsque $T$ est un espace topologique et $\mu$ une mesure de Radon.

Les notations concernant les mesures cylindriques sont celles de [1] et [3]. Pour tout $A \subset T$ on désignera par $\Sigma_{A}$ la trace de la tribu $\Sigma$ sur $A$.

Notre but est la recherche des conditions d'existence d'une densité faible $\vec{f}: T \rightarrow E$ c'est-à-dire d'une fonction $\vec{f}$ scalairement mesurable vérifiant $x^{\prime} \circ \vec{m}=\left(x^{\prime} \circ \vec{f}\right) \cdot \mu$ pour tout $x^{\prime} \in E^{\prime}$, les mesures 
$\vec{m}$ et $\mu$ étant évidemment reliées par la condition: pour tout $A \in \Sigma$ tel que $\mu(A)=0$, on a $\vec{m}(A)=\overrightarrow{0}$ (on note $\vec{m} \ll \mu$ ). On peut raisonnablement supposer que la mesure $\mu$ est concassable (on dit aussi strictement localisable); il existe donc une partition $\left(A_{i}\right)$ de $T$ formée d'éléments de $\Sigma$ vérifiant $\mu\left(A_{i}\right)<+\infty$ de sorte que l'on ait $\mu(B)=$ $\Sigma \mu\left(B \cap A_{i}\right)$ pour tout $B \in \Sigma$. Il est clair que si $\vec{m}_{i}$ et $\mu_{i}$ désignent les restrictions respectives de $\vec{m}$ et $\mu$ à l'ensemble $A_{i}$ alors on a encore $\vec{m}_{i} \ll \mu_{i}$. Par conséquent, pour qu'il existe une densité faible $\vec{f}$ (resp. une densité $\Sigma$-mesurable) il faut et il suffit qu'il en soit ainsi sur chaque ensemble $A_{i}$. On pourra donc supposer, sans restreindre la généralité des résultats, que la mesure $\mu$ est bornée sur $T$.

D'une manière générale, dans la recherche d'une densité (faible ou forte), on est amené pratiquement dans tous les cas à utiliser la propriété du relèvement [10] et [11]. Classiquement, pour que cette méthode s'applique, il est indispensable que le couple $(\vec{m}, \mu)$ vérifie la condition supplémentaire suivante:

$\forall \varepsilon>0$ il existe $A \in \Sigma$ tel que $\mu(T-A) \leqq \varepsilon$ et tel que l'ensemble $S_{A}=\left\{\vec{m}(B) / \mu(B) ; B \in \Sigma_{A}, \mu(B) \neq 0\right\}$ soit borné dans $E$.

Si cette condition est vérifiée, il existe une partition dénombrable $\left(A_{n}\right), n \geqq 1$, de $T$ formée d'éléments de $\Sigma$ de sorte que pour tout $n \geqq 1$ l'ensemble $S_{A_{n}}$ soit borné dans $E$. Mais en vertu de ce qui précède, il suffit de faire l'étude pour tout $n$ du couple $\left(\vec{m}_{n}, \mu_{n}\right)$. En conclusion, on supposera toujours dans tout cet article les conditions suivantes réalisées:

(a) La mesure $\mu$ est une probabilité;

(b) Pour tout $A \in \Sigma$ tel que $\mu(A)=0$, on a $\vec{m}(A)=\overrightarrow{0}$;

(c) L'ensemble $S_{T}=\xi \vec{m}(A) / \mu(A) ; A \in \Sigma, \mu(A) \neq 0$ k est borné dans $E$.

Soit $\alpha$ une mesure cylindrique quelconque sur $E$.

Rappelons la définition suivante (voir [1]).

Definition 1.1. Soit $\alpha$ une mesure cylindrique sur $E, \mathscr{C}$ une topologie sur $E^{\prime}$ et $L: E^{\prime} \rightarrow L^{\circ}(X, \Omega, \eta)$ une fonction aléatoire associée à $\alpha$. On dit que $\alpha$ est d'ordre $p(p \in] 0,+\infty])$ si pour tout $x^{\prime} \in E^{\prime}$ $L\left(x^{\prime}\right) \in L^{p}(X, \Omega, \eta)$. On dit que $\alpha$ est de type $(\mathscr{C}, p)$ si l'application $L$ est continue de $\left(E^{\prime}, \mathscr{C}\right)$ dans $L^{p}(X, \Omega, \eta)$.

Soit $(\vec{m}, \mu)$ le couple introduit ci-dessus; pour tout $x^{\prime} \in E^{\prime}$ on a $x^{\prime} \circ \vec{m} \ll \mu$. Il existe donc une densité scalaire $f_{x^{\prime}} \in L^{1}(T, \Sigma, \mu)$. Il est clair que l'application $L: E^{\prime} \rightarrow L^{\circ}(T, \Sigma, \mu)$ définie par $L\left(x^{\prime}\right)=f_{x^{\prime}}$ est une fonction aléatoire sur $E^{\prime}$. Il lui correspond donc une mesure cylindrique $\lambda$ sur $E$. On dira que $\lambda$ est la mesure cylindrique associée 
au couple $(\vec{m}, \mu)$. On peut déjà donner quelques précisions sur les propriétés de $\lambda$ avec le théorème suivant (qui reproduit un résultat de [20] énoncé pour les espaces de Banach).

THÉORÈmE 1.2. Le couple $(\vec{m}, \mu)$ définit sur $E$ une mesure cylindrique $\lambda$ d'ordre $+\infty$ et de type $\left(\mathscr{C}_{b}, 1\right)$ où $\mathscr{C}_{b}$ désigne la topologie sur $E^{\prime}$ de la convergence uniforme sur les parties bornées de $E$. Si l'espace $E$ est quasi-complet alors $\lambda$ est de type $\left(\mathscr{C}_{c}, 1\right)$, où $\mathscr{C}_{c}$ désigne la topologie sur $E^{\prime}$ de la convergence uniforme sur les disques faiblement compacts de $E$.

Inversement toute mesure cylindrique $\lambda^{\prime}$ sur $E$ (non nécessairement quasi-complet) d'ordre $+\infty$ et de type $\left(\mathscr{C}_{c}, 1\right)$ provient d'un couple $\left(\vec{m}, \mu^{\prime}\right)$ satisfaisant aux conditions (a), (b), (c) énoncées cidessus.

Preuve. Le fait que $\lambda$ soit d'ordre $+\infty$ provient de la condition c). La continuité absolue de $\vec{m}$ par rapport à $\mu$ entraîne que $\lambda$ est de type $\left(\mathscr{C}_{b}, 1\right)$. Dans un espace quasi-complet le rang $\mathscr{R}(\vec{m})$ de la mesure $\vec{m}$ est faiblement relativement compact, donc dans ce cas particulier $\lambda$ est de type $\left(\mathscr{C}_{c}, 1\right)$. Quant à la propriété réciproque du théorème, c'est une simple application du théorème de HahnBanach.

On se propose de retrouver des théorèmes connus du type Radon-Nikodym en utilisant l'outil des mesures cylindriques. Donnons tout d'abord le résultat très simple suivant.

Proposition 1.3. Relativement à un couple $(\vec{m}, \mu)$ il existe toujours une densité $\overrightarrow{f: T \rightarrow E^{\prime \prime}}$ pour dualité ( $\left.E^{\prime \prime}, E^{\prime}\right)$.

Preuve. L'application $L:\left(E^{\prime}, \mathscr{C}_{b}\right) \rightarrow L^{\infty}(T, \Sigma, \mu)$ est continue. Avec [10] il existe une isométrie $I$ de $L^{\infty}(T, \Sigma, \mu)$ dans l'espace $B^{\infty}(T, \Sigma, \mu)$ des fonctions numériques, mesurables et bornées sur $T$. Il suffit alors de poser $\left\langle f(t), x^{\prime}\right\rangle=I\left(L\left(x^{\prime}\right)\right)$ pour tout $x^{\prime} \in E^{\prime}$.

On retrouve alors le résultat suivant de Metivier [16].

THÉORÈME 1.4. Supposons que pour tout $\varepsilon>0$ il existe $A_{\varepsilon} \in \Sigma$ tel que $\mu\left(T-A_{\varepsilon}\right) \leqq \varepsilon$ et $S_{A_{\varepsilon}}$ soit inclus dans un disque faiblement compact de $E$. Alors $i l$ existe une densité faible $\vec{f}$.

Preuve. On se ramène très facilement au cas où $S_{T}$ est lui-même inclus dans un disque compact de $E$. Et tout résulte de (1.3). 
On obtient de même le résultat suivant

THÉORÈmE 1.5. Supposons que E soit semi-réflexif, alors il existe une densité faible $\vec{f}: T \rightarrow E$. Si de plus $E$ est un espace de Fréchet, alors la densité $\vec{f}$ est totalement mesurable.

Preuve. Il est clair avec (1.3) ou (1.4) qu'il existe une densité faible $\vec{f}$. Si $E$ est un espace de Fréchet, l'application. $L:\left(E^{\prime}, \mathscr{C}_{c}\right) \rightarrow$ $L^{\infty}(T, \Sigma, \mu)$ est continue. En étudiant l'application transposée $L^{t}: L^{1}(T, \Sigma, \mu) \rightarrow E$, on établit facilement (voir par exemple [20]) que $\vec{m}$ est à valeurs dans un sous-espace séparable de $E$ et ainsi $\vec{f}$ est totalement mesurable.

Donnons finalement une démonstration du resultat classique suivant:

THÉoRÈme 1.6. Tout dual séparable $E^{\prime}$ d'un espace de Banach $E$ est un espace de Radon-Nikodym.

Preuve. Il est clair que $S_{T}$ est inclus dans un disque $\sigma\left(E^{\prime}, E\right)$ compact. Il existe donc une densité $\vec{f}: T \rightarrow E^{\prime}$ pour la dualité $\left(E^{\prime}, E\right)$. Soit $x$ un point de la boule unité $B^{\prime \prime}$ du bidual $E^{\prime \prime}$. Comme $B^{\prime \prime}$ est $\sigma\left(E^{\prime \prime}, E^{\prime}\right)$ métrisable, il existe une suite $\left(x_{n}\right)$ incluse dans la boule unité $B$ de $E$ qui converge vers $x$ pour la topologie $\sigma\left(E^{\prime \prime}, E^{\prime}\right)$. Ainsi $\langle\vec{f}(t), x\rangle=\lim _{n \rightarrow+\infty}\left\langle\vec{f}(t), x_{n}\right\rangle$, de plus l'ensemble $\left(L\left(x_{n}\right)\right)$ est borné dans $L^{\infty}(T, \Sigma, \mu)$. Il résulte du théorème de Lebesgue que la fonction $x \circ \vec{f}$ est $\mu$-intégrable ce qui suffit.

II. Quelques conditions de concentration cylindrique. Il s'agit de donner des renseignements plus précis que ceux décrits dans le Paragraphe I sur les relations entre le couple $(\vec{m}, \mu)$ et la mesure cylindrique $\lambda$ associée. On désignera par $\mathscr{P}_{f}\left(E^{\prime}\right)$ l'ensemble des parties finies de $E^{\prime}$ et pour toute partie $N=\left\{x_{1}^{\prime}, \cdots, x_{n}^{\prime}\right\} \in \mathscr{P}_{f}\left(E^{\prime}\right)$ on notera par $\Pi_{N}$ l'application de $E$ dans $R^{n}$ définie par $\Pi_{N}(x)=\left(x_{i}^{\prime}(x)\right)$ $1 \leqq i \leqq n$.

Commençons par établir un théorème mettant en évidence l'existence d'un lien étroit entre propriétés des ensembles $S_{A}(A \in \Sigma)$ et les propriétés de concentration cylindrique de la mesure cylindrique $\lambda$.

THÉORÈmE 2.1.

(a) Soit $A$ un élement de la tribu $\Sigma$ tel que $\mu(T \backslash A) \leqq \varepsilon$; alors pour toute partie finie $N \subset E^{\prime}$ on a: 


$$
\lambda\left(\Pi_{N}^{-1} \overline{\left(\Pi_{N}\left(S_{A}\right)\right)}\right) \geqq 1-\varepsilon .
$$

(b) Inversement, soit $C$ un disque de $E$ tel que $\lambda\left(\Pi_{N}^{-1}\left(\overline{\Pi_{N}(C)}\right)\right) \geqq$ $1-\varepsilon$. Alors il existe une partie $A \in \Sigma$ de sorte que l'on ait:

( $\alpha) \mu(T-A) \leqq \varepsilon$;

( $\beta$ ) $S_{A} \subset \bar{C}^{\sigma\left(E, E^{\prime}\right)}$.

Preuve. (a) Soit $N=\left\{x_{i}^{\prime}, \cdots, x_{n}^{\prime}\right\}$ un élément de $\mathscr{P}_{f}\left(E^{\prime}\right)$. Pour tout $x_{i}^{\prime} \in N$ il existe une fonction mesurable $f_{x_{i}^{\prime}}$ partout définie sur $T$ et vérifiant $x_{i}^{\prime} \circ \vec{m}=f_{x_{i}^{\prime}} \cdot \mu$.

Désignons par $f_{N}$ l'application définie sur $T$ par $f_{N}(t)=\left(f_{x_{i}^{\prime}}(t)\right)$ $1 \leqq i \leqq n$. Par définition même de $\lambda$ on a $\lambda\left(\Pi_{N}^{-1} \overline{\left(\Pi_{N}(B)\right)}\right)=$ $\mu\left(f_{N}^{-1}\left(\overline{\Pi_{N}(B)}\right)\right)$. Nous allons établir que $f_{N}^{-1}\left(\overline{\Pi_{N}(B)}\right) \supset A \mu$-presque partout. Supposons le contraire réalisé, alors l'ensemble $P=$ $\left\{t \in A ; f_{N}(t) \notin \overline{\Pi_{N}(B)}\right\}$ est de mesure non nulle. Remarquons que le complémentaire $D$ de $\overline{\Pi_{N}(B)}$ dans $\boldsymbol{R}^{n}$ est réunion dénombrable de pavés $\left.P_{m}=\prod_{i=1}^{n}\right] a_{i}^{m}, b_{i}^{m}\left[\right.$ verifiant: $\bar{P}_{m} \subset D \forall m$. Il existe ainsi un pavé $P_{m_{0}}$ tel que l'ensemble $P \cap f_{N}^{-1}\left(P_{m_{0}}\right)=P^{\prime}$ soit de mesure non nulle. On en tire $\left(\int_{P} f_{x_{i}^{\prime}}(t) d \mu(t) / \mu\left(P^{\prime}\right)\right) \in\left[a_{i}^{m_{0}}, b_{i}^{m_{0}}\right]$ pour tout $1 \leqq i \leqq n$, ainsi $\Pi_{N}\left(\vec{m}\left(P^{\prime}\right) / \mu\left(P^{\prime}\right)\right) \in \bar{P}_{m_{0}} \subset D$. Or l'ensemble $P^{\prime}$ est inclus $A$, ce qui est en contradiction avec l'inclusion ci-dessus.

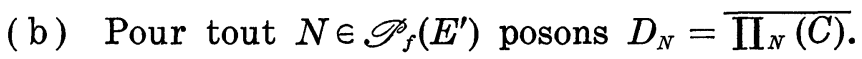

On a comme en 1. $\mu\left(f_{N}^{-1}\left(D_{N}\right)\right)=\lambda\left(\Pi_{N}^{-1}\left(D_{N}\right)\right) \geqq 1-\varepsilon$. Posons $A_{N}=$ $f_{N}^{-1}\left(D_{N}\right)$, la famille $\left(A_{N}\right)\left(N \in \mathscr{P}_{f}\left(E^{\prime}\right)\right)$ est filtrante décroissante. Soit $A$ la borne inférieure latticielle de la famille $\left(A_{N}\right)$ dans le lattice complet $L^{\circ}(T, \Sigma, \mu, \bar{R})$. Il est bien connu qu'il existe une suite $\left(A_{N_{P}}\right) p \in N$ de sorte que $A=\cap A_{N_{P}} \mu . p . p$. , ce qui nous donne $\mu(T-A) \leqq \varepsilon$. Soit maintenant $x^{\prime}$ un point du polaire $C^{\circ} \operatorname{de} C$, alors $f_{x^{\prime}}(t) \in \overline{x^{\prime}(C)} \subset[-1,+1]$ pour $\mu$-presque tout $t \in A$. Il en résulte que pour tout $B \in \sum_{A}$ tel que $\mu(B) \neq 0$ on a $\left\langle\vec{m}(B) / \mu(B), x^{\prime}\right\rangle=$ $\left(\int_{B} f_{x^{\prime}} d \mu / \mu(B)\right) \in[-1,+1]$ et ainsi $S_{A} \subset \bar{C}$.

THÉORÈmE 2.2. La mesure cylindrique $\lambda$ est de Radon sur $\left(E^{\prime \prime}, \sigma\left(E^{\prime \prime}, E^{\prime}\right)\right)$ et elle est portée par l'adhérence $\bar{S}_{T}^{o\left(E^{\prime \prime}, E^{\prime}\right)}$ de $S_{T}$ dans $\left(E^{\prime \prime}, \sigma\left(E^{\prime \prime}, E^{\prime}\right)\right)$.

Preuve. En effet, d'une part $\bar{S}_{T}^{o\left(E^{\prime \prime}, E^{\prime}\right)}$ est un compact dans $\left(E^{\prime \prime}, \sigma\left(E^{\prime \prime}, E^{\prime}\right)\right)$ et d'autre part, avec le Théorème 2.1 , on a $\lambda\left(\Pi_{N}^{-1}\left(\Pi_{N}\left(\bar{S}_{T}\right)\right)\right)=1$. Il suffit d'appliquer le théorème de Prokhorov.

CoROllaire 2.3. Si pour tout $\varepsilon>0$ il existe $A \in \Sigma$ tel que $\mu(T-A) \leqq \varepsilon$ et un compact faible $K \subset E$ tel que $S_{A} \subset K$, alors la mesure cylindrique $\lambda$ est de Radon sur $\left(E, \sigma\left(E, E^{\prime}\right)\right)$. 
On obtient encore comme conséquence de (2.1) le résultat suivant.

THÉORÈmE 2.4. Supposons que pour tout $\varepsilon>0$ il existe un disque faiblement compact $D_{\varepsilon} \subset E$ tel que $\lambda^{*}\left(D_{\varepsilon}\right) \geqq 1-\varepsilon$ ( $\lambda$ est alors de Radon). Dans ces conditions il existe une densité faible $\vec{f}: T \rightarrow E$.

Preuve. Il résulte de la seconde partie du Théorème 2.1 qu'il existe $A \in \Sigma$ de sorte que l'on ait $\mu(T-A) \leqq \varepsilon$ et $S_{A} \subset D_{\varepsilon}$. Il suffit d'appliquer le Théorème 1.4 .

Remarque. On trouvera des variantes de ce résultat dans [3] et [20], dans les deux cas la démonstration s'inspirant d'une idée de Kwapien [13].

J. Pellaumail [17] et indépendamment J. Kupka [12] ont généralisé le théorème de M. Métivier que nous venons d'appliquer ci-dessus sous la forme suivante.

THÉORÈmE 2.5. Si pour tout $\varepsilon>0$ il existe une partie $A \in \Sigma$ et un compact faible $K_{\varepsilon}$ de sorte que l'on ait $\mu(T-A) \leqq$ et $S_{A} \subset K_{\varepsilon}$, alors il existe une densité faible $\vec{f}: T \rightarrow E$.

L'originalité de ce théorème réside dans le fait que, contrairement à (1.4), le compact $K_{\varepsilon}$ n'est pas nécessairement un disque. En fait ce résultat n'est qu'en apparence plus général car on peut facilement se ramener au cas où $K_{\varepsilon}$ est un disque et ceci par la méthode suivante.

Tout d'abord, par un raisonnement décrit dans le Paragraphe I, on peut supposer que $A=T$. Rappelons encore que si la mesure scalaire est atomique alors il est tout à fait évident qu'il existe une densité. Ainsi en décomposant $\mu$ en sa partie diffuse et sa partie atomique, on ramène le problème au cas où $\mu$ est une mesure diffuse. Tout est conséquence du résultat suivant.

Proposition 2.6. On suppose que la mesure $\mu$ est diffuse et qu'il existe un compact faible $K \subset E$ tel que $S_{T} \subset K$. Alors pour tout $\varepsilon>0$ il existe une partie $A \in \Sigma$ et un disque compact $K_{\varepsilon}$ tels que $\mu(T-A) \leqq \varepsilon$ et $S_{A} \subset K_{\varepsilon}$.

Preuve. L'hypothèse $S_{T} \subset K$ implique que le rang $\mathscr{R}(\vec{m})$ de la mesure vectorielle $\vec{m}$ est inclus dans l'enveloppe équilibrée $e(K)$ du compact $K$. L'ensemble $e(K)$ est évidemment lui-même compact; de plus, d'après un résultat de Tweddle [23] généralisant le théorème classique de Liapounov [14], l'adhérence de $\mathscr{R}(\vec{m})$ est un ensemble convexe. Considérons le disque compact $D$ enveloppe convexe de 
$\{\mathscr{R}(\vec{m})\} \cup\{-\mathscr{R}(\vec{m})\}$, alors on a $K \subset \bigcup_{n \geqq 1} n D$. En vertu du Théorème 2.1, $\lambda$ est de Radon sur $\left(E, \sigma\left(E, E^{\prime}\right)\right)$. Pour tout $\varepsilon>0$, il existe donc un entier $n_{\varepsilon}$ de sorte que $\lambda\left(n_{\varepsilon} D\right) \geqq 1-\varepsilon$. On termine avec le Théorème 2.4 .

Remarque. Dans [8] D. J. Hebert Jr. donne un théorème du type Radon-Nikodym avec les mêmes conditions que dans (2.5) en rajoutant l'hypothèse que les compacts $K_{\varepsilon}$ sont métrisables, ce qui est tout à fait inutile. De plus, pour démontrer son résultat, Hebert utilise un théorème de décomposition établi par lui-même dans [7] dont la démonstration nous semble fausse. En effet, il affirme que lorsque tous les compacts de $E$ sont métrisables alors la tribu cylindrique contient les parties compactes, ce qui est généralement inexact. Ce dernier point nous a été signalé par M. W. Schachermayer.

Le cas particulier des espaces sousliniens quasi-complets. Commençons par rappeler quelques propriétés fondamentales des espaces sousliniens (on pourra consulter par exemple [1]).

A. Soit $E$ un espace souslinien (localement convexe séparé) alors la tribu borélienne $\mathscr{B}$ de $E$ coincide avec la tribu cylindrique $\sigma \mathscr{C}$.

B. Toute mesure borélienne sur $E$ est une mesure de Radon.

C. Tout compact $K$ de $E$ est métrisable.

Ces propriétés particulières des espaces sousliniens permettent de répondre d'une manière très précise au problème de l'existence d'une densité.

THÉoRÈmE 2.8. Soit $E$ un espace souslinien quasi-complet, les assertions suivantes sont équivalentes:

(a) La mesure cylindrique $\lambda$ est de Radon sur $E$;

(b) La mesure cylindrique $\lambda$ est de Radon sur $\left(E, \sigma\left(E, E^{\prime}\right)\right)$;

(c) Il existe une densité faible $\vec{f}: T \rightarrow E$;

(d) Il existe une densité $\Sigma$-mesurable unique $\vec{f}: T \rightarrow E$.

(e) Pour tout $\varepsilon>0$ il existe $A \in \Sigma$ et un compact (resp. un compact faible) $K_{\varepsilon}$ tels que l'on ait: $\mu(T-A) \leqq \varepsilon$ et $S_{A} \subset K_{\varepsilon}$.

Preuve. Si $\mathscr{C}_{1}$ et $\mathscr{C}_{2}$ sont deux topologies sousliniennes comparables, elles sont Radon-équivalentes, ce qui prouve $(a) \Leftrightarrow(b)$. Il résulte des points $\mathrm{A}$ et $\mathrm{B}$ cités ci-dessus que s'il existe une densité faible $\overrightarrow{f:} T \rightarrow E$ alors $\lambda=\vec{f}(\mu)$ est une mesure borélienne, donc de Radon, ce qui montre $(\mathrm{c}) \Longrightarrow(\mathrm{a})$. L'implication $(\mathrm{c}) \Leftrightarrow(\mathrm{d})$ résulte $\mathrm{du}$ point A.

Montrons que l'on a $(\mathrm{a}) \Rightarrow(\mathrm{c})$ : la mesure $\lambda$ étant de Radon sur $E$, il existe une suite $\left(K_{n}\right)$ de compacts métrisables et un ensemble négligeable $N$ de sorte que $E=N \cup\left(\cup K_{n}\right)$. Le résultat est consé- 
quence du Théorème 2.4.

(a) $\Leftrightarrow(\mathrm{e})$ : c'est un corollaire du Théorème 2.1.

Remarque. Toute fonction $\vec{f}: T \rightarrow E m$-sommable au sens de E. Thomas [22] (ou intégrable au sens de Pettis) définit un couple $(\vec{m}, \mu)$ de telle sorte que $\vec{f}$ en est la densité faible. Il est alors très facile de retrouver, par l'intermédiaire de la mesure $\lambda$ associée, certains résultats de E. Thomas [22] sur les fonctions $m$-sommables à valeurs dans les elc sousliniens et quasi-complets.

A titre d'exemple, demontrons le Théorème 5 du Paragraphe 3 de [22].

ThÉonème 2.9 (E. Thomas). Soient $E$ et $\widetilde{E}$ deux espaces quasicomplets avec $E$ espace de Souslin. On suppose que $E$ est un sousespace vectoriel de $\widetilde{E}$ et que l'injection canonique $j: E \rightarrow \widetilde{E}$ est continue. Soit $\vec{f}: T \rightarrow E$ une fonction vectorielle à valurs dans $E$, posons $\vec{f}^{\prime}=j \circ \vec{f}$. La fonction $\vec{f}$ est m-sommable si et seulement si $\vec{f}^{\prime}$ est m-sommable et pour tout $A \in \Sigma$ on $a \int_{A} \vec{f}^{\prime} d \mu \in E$.

Preuve. Le point difficile c'est de démontrer que la condition est suffisante. On a besoin pour cela du résultat du type OrliczPettis suivant (cité par E. Thomas dans [22]; on pourra consulter également [6] ou [5]):

LEMme. Soient $\mathscr{C}_{1}$ et $\mathscr{C}_{2}$ deux topologies sousliniennes sur un elc $E$. On suppose que $\mathscr{C}_{1}$ est moins fine que $\mathscr{C}_{2}$ et que l'espace $\left(E, \mathscr{C}_{1}\right)$ est quasi-complet. Alors les espaces $\left(E, \mathscr{C}_{1}\right)$ et $\left(E, \mathscr{C}_{2}\right)$ ont les mêmes sous-séries convergentes [6].

Revenons à la preuve de (2.9); l'application $\vec{m}: \Sigma \rightarrow E$ définie par $\vec{m}(A)=\int_{A} \vec{f}^{\prime} d \mu$ est une mesure vectorielle à valeurs dans $E$ muni de la topologie $\mathscr{C}_{2}$ induite par celle de $\widetilde{E}$. C'est donc en vertu du lemme une mesure à valeurs dans $E$ muni de sa topologie initiale $\mathscr{C}_{1}$. La mesure cylindrique $\lambda$ associée est de Radon $\operatorname{sur}\left(E, \mathscr{C}_{2}\right)$ (lorsque $\vec{m}$ est à valeurs dans $\left(E, \mathscr{C}_{2}\right)$ ); de plus, elle est scalairement concentrée sur les disques compacts de $\left(E, \mathscr{C}_{1}\right)$ (lorsque $\vec{m}$ est prise à valeurs dans $\left(E, \mathscr{C}_{1}\right)$ ). Ceci prouve que $\lambda$ est de Radon sur $E$ muni de sa topologie initiale $\mathscr{C}_{1}$, donc avec (2.4) $\vec{f}$ est une densité à valeurs dans $E$.

On dit qu'un elc $E$ est un espace de Radon-Nikodym (resp. Radon-Nikodym faible) lorsque tout couple $(\vec{m}, \mu)$ admet une densité $\vec{f} \Sigma$-mesurable (resp. une densité faible). Pour un espace souslinien ces deux notions se confondent. Donnons quelques propriétés de 
stabilité de la classe des espaces de Radon-Nikodym sousliniens.

THÉORÈme 2.10. Soit $E$ un espace de Radon-Nikodym souslinien (non nécessairement quasi-complet). Tout sous-espace fermé $F$ de $E$ est un espace de Radon-Nikodym (souslinien).

Preuve. Soit $\vec{m}: \Sigma \rightarrow F$ une mesure vectorielle à valeurs dans $F$. Déjà il existe une densité $\vec{f}$ à valeurs dans $E$ et la mesure $\lambda$ associée à $(\vec{m}, \mu)$ est de Radon sur $E$. D'après le Théorème 2.1 elle est portée par $F$, ainsi on a $\mu\left(\vec{f}^{-1}(E \backslash F)\right)=\lambda(E \backslash F)=0$ ce qui suffit. La même méthode permet de démontrer le résultat suivant.

THÉorÈme 2.11. Soit E un espace de Radon-Nikodym souslinien. Alors tout sous-espace souslinien $F \subset E$ de codimension dénombrable dans $E$ est un espace de Radon-Nikodym.

Preuve. Considérons un couple $(\vec{m}, \mu)$ où $\vec{m}: \Sigma \rightarrow F$ est à valeurs dans $F$. Soit $G$ un supplémentaire algébrique de $F$. Il existe une densité $\vec{f}: T \rightarrow E$ et la mesure $\lambda=\vec{f}(\mu)$ est de Radon sur $E$. Elle est donc encore de Radon sur l'espace souslinien $F \oplus G$ somme directe topologique de $F$ et $G$, ce qui nous ramène aux hypothèses du Théorème 2.10 .

Remarque. Si dans un elc $E$ il existe un sous-espace $F$ souslinien de codimension dénombrable dans $E$ alors l'espace $E$ est lui-même nécessairement souslinien.

III. Concentration cylindrique et $\sigma$-dentabilite dans les elc quasi-complets. Commençons par préciser quelque peu le lien entrevu dans (2.1) entre les ensembles $S_{A}(A \in \Sigma)$ et la mesure cylindrique $\lambda$.

THÉORÈme 3.1. Soit $E$ un espace localement convexe, les assertions suivantes sont equivalentes:

(a) Pour tout $\varepsilon>0$ et pour tout $V$ voisinage de zéro dans $E$, $i l$ existe un compact $K_{V}^{\varepsilon} \subset E$, tel que $\lambda^{*}\left(K_{V}^{\varepsilon}+V\right) \geqq 1-\varepsilon$.

(b) Comme (a) avec un sous-espace vectoriel de dimension finie $G_{V}^{\varepsilon} \subset E$ à la place de $K_{V}^{\varepsilon}$.

(c) Pour tout $\varepsilon>0$ et pour tout $V$ voisinage de zéro dans $E$, $i l$ existe $A \in \Sigma$ et un compact $K_{V}^{s} \subset E$, tels que $\mu(T-A) \leqq \varepsilon$ et $S_{A} \subset K_{V}^{\mathrm{\varepsilon}}+V$.

(d) Comme (c) avec un sous-espace vectoriel de dimension finie $G_{V}^{\varepsilon} \subset E$ à la place de $K_{V}^{\varepsilon}$. 
Preuve. On a l'équivalence $(\mathrm{a}) \Leftrightarrow(\mathrm{b})$ pour toute mesure cylindrique sur $E$ car tout espace localement convexe vérifie la propriété (P) de [3].

(a) $\Leftrightarrow$ (c) On se ramène facilement à supposer que $K_{V}^{\varepsilon}$ est un disque et $V$ un disque fermé, on est donc dans le domaine d'application du Théorème 2.1.

$(b) \Leftrightarrow(d) \quad$ C'est le même démonstration que celle de l'équivalence précédente.

Remarque. La condition (a) du Théorème 3.1 n'est rien d'autre que la condition (B) dont nous avons fait mention dans l'introduction.

Le résultant que nous venons d'établir peut encore s'améliorer. En effet, on peut énoncer:

ThÉorème 3.2. Soit $E$ un espace localement convexe, les assertions suivantes sont équivalentes:

(a) Pour tout $\varepsilon>0$ et pour tout $V$ voisinage de zéro dans $E$, il existe $A \in \Sigma$ et un compact $K_{V}^{\varepsilon} \subset E$, tels que l'on ait $\mu(T-A) \subset \varepsilon$ et $S_{A} \subset K_{V}^{\varepsilon}+V$.

(b) Pour tout ensemble $A \in \Sigma$ de mesure non nulle et pour tout $V$ voisinage de zéro dans $E$, il existe $B \in \Sigma$ et un compact $K_{B} \subset E$, tels que l'on ait $S_{B} \subset K_{B}+V$ avec $B$ de mesure non nulle et inclus dans $A$.

(c) Comme (a) avec un sous-espace vectoriel de dimension finie $G_{V}^{\varepsilon} \subset E$ à la place de $K_{V}^{\varepsilon}$.

(d) Comme (b) avec un sous-espace vectoriel de dimension finie $G_{B} \subset E$ à la place de $K_{B}$.

\section{Preuve.}

(a) $\Leftrightarrow$ (b) Soit $A$ un élément de $\Sigma$ de mesure non nulle. Il existe $A^{\prime} \in \Sigma$ et un compact $K \subset E$, tels que l'on ait $\mu\left(T-A^{\prime}\right) \leqq(1 / 2) \mu(A)$ d'ume part et $S_{A^{\prime}} \subset K+V$. Posons $B=A \cap A^{\prime}$, alors $S_{B} \subset S_{A^{\prime}} \subset K+V$ et d'autre part

$$
\mu(B)=\mu(A)-\mu(A \backslash B) \geqq \mu(A)-\frac{1}{2} \mu(A)>0 .
$$

(b) $\Leftrightarrow$ (a) Soit $V$ un voisinage de zéro dans $E$. Avec l'axiome du choix, il existe une suite $\left(A_{n}\right)_{n \geqq 1}$ d'éléments de $\Sigma$ deux à deux disjoints vérifiant:

( $\alpha) \cup A_{n}=T \mu . p . p$. et l'on a $\mu\left(A_{n}\right)>0$ pour tout $n \geqq 1$.

$(\beta)$ A chaque ensemble $A_{n}$ correspond un compact $K_{n} \subset E$ (que l'on peut toujours supposer disqué), de sorte que $S_{A_{n}} \subset K_{n}+V$ pour tout $n \geqq 1$. 
Soit maintenant $\varepsilon>0$ : il existe un entier $N \geqq 1$ tel que l'on ait $\mu\left(T \backslash \bigcup_{1}^{N} A_{n}\right) \leqq \varepsilon$. Posons $A=\bigcup_{1}^{N} A_{n}$ et soit $K=\Gamma\left(\bigcup_{1}^{N} K_{n}\right)$ l'enveloppe disquée de la suite $\left(K_{n}\right)_{1 \leq n \leq N}$. L'ensemble $K$ est un disque compact et il est facile de vérifier que $S_{A} \subset K+V$.

(c) $\Leftrightarrow(d)$ : C'est la même méthode de démonstration que celle décrite précédemment.

(a) $\Leftrightarrow$ (c): Ce point est démontré en (3.1).

Il est facile de vérifier que lorsqu'on remplace le compact $K$ de (3.1) (resp. de (3.2)) par un disque faiblement compact alors on a encore $(a) \Leftrightarrow(c)$ comme dans (3.1) (resp. (a) $\Leftrightarrow(b)$ comme dans (3.2)). Pour plus de clarté, isolons cette propriété sous la forme de théorème.

THÉORÈme 3.3. Soit $E$ un elc, les assertions suivantes sont équivalentes:

(a) Pour tout $\varepsilon>0$ et pour tout $V$ voisinage de zéro dans $E$, il existe un ensemble $A \in \Sigma$ et un disque faiblement compact $D_{V}^{\varepsilon} \subset E$, tels que l'on ait $\mu(T-A) \leqq \varepsilon$ et $S_{A} \subset D_{V}^{\varepsilon}+V$.

(b) Pour tout ensemble $A \in \Sigma$ de mesure non nulle et pour tout $V$ voisinage de zéro dans $E$, il existe un ensemble $B \subset A, B \in \Sigma$ de mesure non nulle et un disque faiblement compact $D_{B} \subset E$, tels que l'on ait $S_{B} \subset D_{B}+V$.

(c) Pour tout $\varepsilon>0$ et pour tout $V$ voisinage de zéro dans $E$, $i l$ existe un disque faiblement compact $D_{V}^{\varepsilon} \subset E$, tel que $\lambda^{*}\left(D_{V}^{\varepsilon}+V\right) \geqq$ $1-\varepsilon$.

Remarque. Si $E$ est un "bon espace" [3] alors le Théorème 3.1 (resp. le Théorème 3.2) donne des conditions nécessaires et suffisantes pour que la mesure $\lambda$ soit de Radon sur $E$.

Definition 3.4. Soit $E$ un espace localement convexe séparé. $S i$ pour toute mesure cylindrique $\alpha$ sur $E$ les conditions qui suivent sont équivalentes, on dira que $E$ est un $B-F$ espace:

(a) La mesure $\alpha$ est de Radon sur $\left(E, \sigma\left(E, E^{\prime}\right)\right)$;

(b) Pour tout $\varepsilon>0$ et pour tout $V$ voisinage de zéro dans $E$, il existe un compact faible $D_{V}^{\varepsilon} \subset E$, tel que l'on ait $\alpha^{*}\left(D_{V}^{\varepsilon}+V\right) \geqq$ $1-\varepsilon$.

La théorie des "bons espaces" repose sur un résultat de De Acosta (Lemme (IV, 2, 1) de [3]). On peut encore montrer que ce résultat est valable lorsque'on remplace mot pour mot compact par faiblement compact, ce qui permet de donner des conditions nécessaires et suffisantes pour que $\alpha$ soit de Radon sur $\left(E, \sigma\left(E, E^{\prime}\right)\right)$. Par exemple on le résultat suivant (analogue au Théorème $(I V, 2,1)$ de [3]). 
THÉORÈME 3.5. Soit E un elc complet et soit $\alpha$ mesure cylindrique sur $E$. Les assertions suivantes sont équivalentes:

(a) La mesure $\alpha$ est de Radon sur $\left(E, \sigma\left(E, E^{\prime}\right)\right)$;

(b) Soit $\mathscr{U}$ un système fondamental de voisinages disqués de zéro dans E. Pour tout $\varepsilon>0$, et pour tout $V \in \mathscr{Q}$, il existe un compact faible $K_{V}^{\varepsilon} \subset E$, tel que l'on ait $\alpha^{*}\left(\bigcap_{V \in F}\left(K_{V}^{\varepsilon}+V\right)\right) \geqq 1-\varepsilon$ pour toute partie finie $\mathscr{T} \subset \mathscr{Q}$.

Nous n'avons pas l'intention de faire une étude systématique des $B-F$ espaces. Contentons-nous de remarquer, que si un $B-F$-espace $E$ est Radon-équivalent à son affaibli $\left(E, \sigma\left(E, E^{\prime}\right)\right)$, alors c'est également un "bon espace." On peut montrer facilement que tout espace de Fréchet est un $B-F$ espace.

Liaison avec la $\sigma$-dentabilité. Considérons un couple $(\vec{m}, \mu)$ où $\vec{m}: \Sigma \rightarrow E$ est une mesure vectorielle à valeurs dans un espace de Banach. Le résultat classique de Rieffel [18] assure que si tout ensemble $A \in \Sigma$ de mesure non nulle contient un élément $B \in \Sigma$ également de mesure non nulle et tel que $S_{B}$ soit $\sigma$-dentable, alors le couple $(\vec{m}, \mu)$ est densitable. Nous allons montrer que ce résultat peut s'améliorer, et ceci en introduisant une notion plus générale de $\sigma$-dentabilité.

Pour toute partie bornée $D \subset E$ on désignera par $\sigma(D)$ l'ensemble des sommes $\Sigma \lambda_{n} x_{n}$ supposées convergentes construites à partir de points $x_{n} \in D$ et de scalaires $\lambda_{n}>0$ tels que $\Sigma \lambda_{n}=1$.

Introduisons les définitions suivantes:

Definition 3.6. Soit $\mathscr{F}$ une famille de parties de $E$. Un sous-ensemble $A \subset E$ sera dit $\mathscr{F}$ - $\sigma$-dentable si pour tout voisinage de zéro $V$ de $E$ il existe un point $x \in A$ et un ensemble $B \in \mathscr{F}$ tels $q u e x \notin \sigma(A \backslash(B+V))$.

Plus particulièrement, lorsque $\mathscr{F}$ est constituée de parties finies, on parlera de parties $\sigma$-dentables; lorsqu'on prend pour $\mathscr{F}$ l'ensemble des sous-espaces vectoriels de dimension finie de $E$ on dira que $A$ est linéairement $\sigma$-dentable; finalement on parlera de parties $K-\sigma$ dentables si $\mathscr{F}$ coincide avec la famille des parties faiblement compactes de $E$.

Il est clair que toute partie $\sigma$-dentable est linéairement $\sigma$-dentable, et que si $E$ est normé, toute partie bornée linéairement $\sigma$-dentable est $K$ - $\sigma$-dentable.

Le théorème qui suit justifie l'introduction de ces notions. 
THÉORÈmE 3.7. Relativement à un couple $(\vec{m}, \mu)$, avec $\vec{m}: \Sigma \rightarrow E$, supposons la condition suivante réalisée:

Pour tout $A \in \Sigma, \mu(A)>0$, il existe $B \in \Sigma$ inclus dans $A$ et de mesure non nulle tel que $S_{B}$ soit linéairement $\sigma$-dentable (resp. $K$ - $\sigma$ dentable).

Alors pour tout $A \in \Sigma, \mu(A)>0$, il existe $B \in \Sigma$ inclus dans $A$ et de mesure non nulle et un sous-espace vectoriel de dimension finie $F \subset E$ (resp. un compact faible $K \subset E$ ) tels que l'on ait $S_{B} \subset F+V\left(\right.$ resp. $\left.S_{B} \subset K+V\right)$.

Preuve. Soit donc $A \in \Sigma, \mu(A)>0$, il existe $B \in \Sigma, B \subset A, \mu(B)>0$ tel que $S_{B}$ soit linéairement $\sigma$-dentable (resp. $K$ - $\sigma$-dentable). Ainsi, $V$ étant donné, il existe un point $x \in S_{B}$, et un sous-espace vectoriel $F \subset E$ (resp. un compact faible $K \subset E$ ) tels que $x \notin \sigma\left(S_{B} \backslash(F+V)\right.$ ) (resp. $x \notin \sigma\left(S_{B} \mid(K+V)\right)$ ). On peut écrire $x=\vec{m}\left(B_{0}\right) / \mu\left(B_{0}\right)$, avec $B_{0} \in \Sigma, B_{0} \subset B$ et $\mu\left(B_{0}\right)>0$. Alors, ou bien il existe $B^{\prime} \subset B_{0}, B^{\prime} \in \Sigma$, $\mu\left(B^{\prime}\right)>0$ tel que $S_{B^{\prime}} \subset F+V$ (resp. $S_{B^{\prime}} \subset K+V$ ) ou, dans le cas contraire, il existe avec l'axiome du choix une partition $\left(B_{n}^{\prime}\right)$ de $B_{0}$ constituée d'ensembles $B_{n}^{\prime} \in \Sigma$ de mesure non nulle et vérifiant $\vec{m}\left(B_{n}^{\prime}\right) / \mu\left(B_{n}^{\prime}\right) \notin F+V\left(\right.$ resp. $\left.\vec{m}\left(B_{n}^{\prime}\right) / \mu\left(B_{n}^{\prime}\right) \notin K+V\right)$. Ainsi le point $x=\vec{m}\left(B_{0}\right) / \mu\left(B_{0}\right)$ s'écrit

$$
x=\sum_{1}^{+\infty} \frac{\vec{m}\left(B_{n}^{\prime}\right)}{\mu\left(B_{n}^{\prime}\right)} \times \frac{\mu\left(B_{n}^{\prime}\right)}{\mu\left(B_{0}\right)}
$$

et on obtient une contradiction.

Comme application du Théorème 3.7 on obtient, dans le cas des espaces de Banach, le résultat suivant (généralisant un théorème classique de Rieffel [18]).

THÉORÈme 3.8. Soit $E$ un espace de Banach, on suppose la condition du Théorème 3.7 réalisée. Alors il existe une densité $\vec{f}$ totalement mesurable.

Preuve. Il suffit de construire, pour tout $\varepsilon>0$, par récurrence, une suite $\left(A_{n}\right)$ d'éléments de $\Sigma$ et une suite $\left(K_{n}\right)$ de disques faiblement compacts de $E$ vérifiant.

(a) $\mu\left(T-A_{1}\right) \leqq \varepsilon 2^{-1}$.

(b) $A_{n+1} \subset A_{n}$ et $\mu\left(A_{n}-A_{n+1}\right) \leqq \varepsilon 2^{-(n+1)}$;

(c) $S_{A_{n}} \subset K_{n}+B(0,1 / n)$ où $B(0,1 / n)$ désigne la boule de rayon $1 / n$ du Banach $E$.

Posons $A=\bigcap A_{n}$ et $K=\bigcap\left(K_{n}+B(0,1 / n)\right)$, alors $K$ est un disque compact et l'on a $\mu(T-A) \leqq \varepsilon$ et $S_{A} \subset K$, ce qui termine.

H. Maynard [15] a démontré en 1973 qu'un espace de Banach $E$ est 
un espace de Radon-Nikodym si et seulement si tout borné de $E$ est $\sigma$-dentable (au sens de [18]). Ce résultat se généralise agréablement au cas des espaces quasi-complets sous la forme suivante.

THÉorème 3.9. Soit $E$ un elc quasi-complet, les assertions suivantes sont équivalentes:

(a) Tout borné $B$ de $E$ est $\sigma$-dentable,

(b) Tout borné $B$ de $E$ est linéairement $\sigma$-dentable,

(c) Toute mesure 入 provenant d'un couple $(\vec{m}, \mu)$ vérifie la condition (B). Si de plus $E$ est un "bon espace," ces conditions sont équivalentes à:

(d) Toute mesure $\lambda$ provenant d'un couple $(\vec{m}, \mu)$ est de Radon sur $E$.

Preuve. Il est clair que l'on a: $(\mathrm{a}) \Rightarrow(\mathrm{b}) \Rightarrow(\mathrm{c}) \Leftrightarrow(\mathrm{d})$. Le point délicat c'est la démonstration de l'implication $(\mathrm{c}) \Rightarrow(\mathrm{a})$. Partant du fait que $E$ possède un borné $D$ non $\sigma$-dentable, il faut mettre à défaut la condition (B). Les idées de R. E. Huff [9] et H. Maynard [15] vont nous guider dans cette démarche. Considérons pour cela l'intervalle $T=[0,1$, et soit $\mu$ la mesure de Lebesgue sur $T$. Il existe une suite $\pi_{n}=\left(A_{p}^{n}\right)_{n \geqq 1, p \geqq 1}$ de partitions dénombrables de l'intervalle $T$, une suite $\left(x_{p}^{n}\right)_{n \geqq 1, p \geqq 1}$ de points de $E$, une suite $\left(\varepsilon_{p}^{n}\right)_{n \geqq 1, p \geqq 1}$ de nombres positifs non nuls et un voisinage $V$ de zéro dans $E$, vérifiant les points suivants:

(a) Pour tout $n \geqq 1$, on a $\pi_{n+1} \geqq \pi_{n}\left(\pi_{n+1}\right.$ est plus fine que $\left.\pi_{n}\right)$; de plus, chaque ensemble $A_{p}^{n}$ est un intervalle de la forme $A_{p}^{n}=\left[\alpha_{p}^{n}, \beta_{p}^{n}[\right.$.

(b) Pour tout double indice $(n, p)$ notons par $k(n, p)$ l'ensemble des entiers $q \geqq 1$ tels que l'on ait $A_{q}^{n+1} \subset A_{p}^{n}$. On a alors $A_{p}^{n}=$ $\mathrm{U}_{q \in k(n, p)} A_{q}^{n+1}$.

(c) Pour tout couple $(n, p)$ on a $\sum_{q \in k(n, p)} \varepsilon_{q}^{n+1}=\varepsilon_{p}^{n}$, et l'entier $n \geqq 1$ étant fixé on a $\sum_{p \geqq 1} \varepsilon_{p}^{n}=1$.

(d) Pour tout couple $(n, p)$ on a $\varepsilon_{p}^{n} x_{p}^{n}=\sum_{q \in k(n, p)} \varepsilon_{q}^{n+1} x_{q}^{n+1}$.

(e) Pout tout couple $(n, p)$ notons par $K(n, p)$ l'ensemble des couples d'indices $(r, s)$ vérifiant $A_{s}^{r} \supset A_{p}^{n}$, on a alors

$$
x_{q}^{n+1} \in D \backslash\left(\left\{x_{s}^{r} ;(r, s) \in K(n, p)\right\}+V\right)
$$

pour tout

$$
q \in k(n, p) \text {. }
$$

Passons à la construction explicite. Soit $x_{0}$ un point de $D$; comme $D$ n'est pas $\sigma$-dentable, il existe un voisinage $V$ de zéro dans $E$ tel que $x_{0} \in \sigma(D \backslash V)$. Il en résulte que $x_{0}$ est de la forme $x_{0}=\sum_{p=1}^{+\infty} \varepsilon_{p}^{1} x_{p}^{1}$ avec $\varepsilon_{p}^{1}>0 \forall p, \Sigma \varepsilon_{p}^{1}=1$ et $x_{p}^{1}=D \backslash V$ pour tout $p \geqq 1$. 
Posons $A_{1}^{1}=\left[0, \varepsilon_{p}^{1}\left[\right.\right.$ et $A_{p+1}^{1}=\left[\varepsilon_{1}^{1}+\cdots+\varepsilon_{p}^{1}, \varepsilon_{1}^{1}+\cdots+\varepsilon_{p}^{1}+\varepsilon_{p+1}^{1}[\right.$ pour tout $p \geqq 1$. Les éléments $\left(A_{p}^{1}\right)_{p \geqq 1}$ constituent la partition $\pi_{1}$. Supposons la construction réalisée jusqu'au degré $n>1$. Fixons un point $x_{p}^{n}$, l'ensemble $D$ n'étant pas $\sigma$-dentable, on a

$$
\varepsilon_{p}^{n} x_{p}^{n}=\sum_{q=1}^{+\infty} \varepsilon_{p, q}^{n+1} x_{p, q}^{n+1} \quad \text { avec } \quad \varepsilon_{p}^{n}=\sum_{q=1}^{+\infty} \varepsilon_{p, q}^{n+1}\left(\varepsilon_{p, q}^{n+1}>0\right)
$$

et $x_{p, q}^{n+1} \in D \backslash\left(\left\{x_{s}^{r} ;(r, s) \in K(n, p)\right\}+V\right)$. Décomposons l'intervalle $A_{p}^{n}$ en une suite d'intervalles consécutifs $A_{p, q}^{n+1}$ (de la forme $[\alpha, \beta[$ ) de longueurs égales respectivement à $\varepsilon_{p, q}^{n+1}$. En réunissant les ensembles $\left(A_{p, q}^{n+1}\right)_{p \geqq 1, q \geqq 1}$, les points $\left(x_{p, q}^{n+1}\right)_{p \geqq 1, q \geqq 1}$, les nombres $\left(\varepsilon_{p, q}^{n+1}\right)_{p \geqq 1, q \geqq 1}$, et en renumérotant, on obtient des suites vérifiant les conditions (a), (b), (c), (d), (e) relatives au degré $n+1$. Désignons maintenant par $\sum_{n}$ la tribu engendrée par $\pi_{n}$ et par $\Sigma$ la tribu engendrée par l'anneau $\mathscr{R}=\cup \sum_{n}$. Considérons l'application $\vec{m}_{0}: \mathscr{R} \rightarrow E$ définie par $\vec{m}_{0}\left(A_{p}^{n}\right)=\varepsilon_{p}^{n} x_{p}^{n}$; elle se prolonge en une mesure vectorielle $\vec{m}: \Sigma \rightarrow E$. En effet, posons $\vec{f}_{n}=\sum_{p \geqq 1} x_{p}^{n} 1_{A_{p}^{n}}$ et soit $\vec{m}_{n}$ la mesure vectorielle sur $\Sigma$ définie par $\vec{m}_{n}(A)=\int_{A} \vec{f}_{n} d \mu$ pour tout $A \in \Sigma$. Fixons $A \in \Sigma$, il est facile de vérifier (en approchant $A$ par des ensembles $B \in \mathscr{R}$ ) que la suite $\left(\vec{m}_{n}(A)\right)_{n \geqq 1}$ est de Cauchy dans $E$. Comme $E$ est quasi-complet elle converge; posons $\vec{m}(A)=\lim _{n \rightarrow++\infty} \vec{m}_{n}(A)$, il résulte du théorème classique de Nikodym que $\bar{m}$ est une mesure vectorielle sur $\Sigma$ prolongeant $\vec{m}_{0}$.

Notons par $P$ la jauge de $V$; il existe une constante $K>0$ telle que l'on ait $P(\vec{m}(A)-\vec{m}(B)) \leqq K \mu(A \Delta B)$ pour tout couple $A, B \in \Sigma$. Montrons maintenant que le couple $(\vec{m}, \mu)$ ne vérifie pas la condition (B). Fixons pour cela $B \in \Sigma, \mu(B)>0$, et $\varepsilon>0$; il existe un entier $n_{0} \geqq 1$ et une suite $\left(A_{p}^{n_{0}}\right)_{p \in S_{0}}$ de sorte que $\bigcup_{p \in S_{0}} A_{p}^{n_{0}} \supset B$ et $\mu\left(\left(\mathrm{U}_{p \in S_{0}} A_{p}^{n_{0}}\right) \backslash B\right) \leqq \varepsilon \mu(B)$. On en tire facilement qu'il existe un entier $p_{0} \in S_{0}$ tel que l'on ait $\mu\left(A_{p_{0}}^{n_{0}} \mid\left(A_{p_{0}}^{n_{0}} \cap B\right)\right) \leqq \varepsilon \mu\left(A_{p_{0}}^{n_{0}} \cap B\right)$. Posons $A_{p_{0}}^{n_{0}}=C_{0}$ et $A_{p_{0}}^{n_{0}} \cap B=B_{0}$, on a alors

$$
\begin{aligned}
& P\left(\frac{\vec{m}\left(C_{0}\right)}{\mu\left(C_{0}\right)}-\frac{\vec{m}\left(B_{0}\right)}{\mu\left(B_{0}\right)}\right) \leqq \frac{1}{\mu\left(B_{0}\right)+\varepsilon^{\prime}} \cdot P\left(\vec{m}\left(B_{0}\right)-\vec{m}\left(C_{0}\right)\right. \\
& \quad+\frac{\varepsilon^{\prime}}{\left(\mu\left(B_{0}\right)+\varepsilon^{\prime}\right) \mu\left(B_{0}\right)} \cdot P\left(\vec{m}\left(B_{0}\right)\right) \leqq K\left(\frac{\varepsilon \mu\left(B_{0}\right)}{\mu\left(B_{0}\right)+\varepsilon^{\prime}}+\frac{\varepsilon^{\prime} \mu\left(B_{0}\right)}{\mu\left(B_{0}\right)+\varepsilon^{\prime}}\right) \leqq K \varepsilon,
\end{aligned}
$$

où la constante $\varepsilon^{\prime}$ est évidemment définie par $\varepsilon^{\prime}=\mu\left(C_{0}\right)-\mu\left(B_{0}\right) \leqq \varepsilon$. Avec le choix $\varepsilon=(1 / 3 K)$ on a donc $P\left(\vec{m}\left(C_{0}\right) / \mu\left(C_{0}\right)-\vec{m}\left(B_{0}\right) / \mu\left(B_{0}\right)\right) \leqq 1 / 3$. On réitère la construction en prenant $B_{0}$ à la place de $B$ et, ainsi de suite par récurrence. Ce procédé permet d'obtenir une suite décroissante $\left(C_{n}\right)_{n \geqq 0}$ d'éléments de la forme $A_{q}^{p}$, et une suite décroissante $\left(B_{n}\right)_{n \geqq 0}$ d'éléments de $\Sigma$ vérifiant: $P\left(\vec{m}\left(C_{n}\right) / \mu\left(C_{n}\right)-\vec{m}\left(B_{n}\right) / \mu\left(B_{n}\right)\right) \leqq 1 / 3$. Mais il résulte de la condition (e) ci-dessus que pour tout couple 
d'entiers $n \neq n^{\prime}$ on a $P\left(\vec{m}\left(C_{n}\right) / \mu\left(C_{n}\right)-\vec{m}\left(C_{n^{\prime}}\right) / \mu\left(C_{n^{\prime}}\right)\right) \geqq 1$. Ces deux inégalités impliquent que pour tout couple d'entiers $n \neq n^{\prime}$ on a $P\left(\vec{m}\left(B_{n}\right) / \mu\left(B_{n}\right)-\vec{m}\left(B_{n^{\prime}}\right) / \mu\left(B_{n^{\prime}}\right)\right) \geqq 1 / 3$. En conclusion on a établi que l'ensemble $S_{B}$ n'est pas précompact dans $(E, P)$. Cette propriété étant indépendante du choix de $B \in \Sigma, \mu(B) \neq 0$, elle contredit manifestement le point $(b)$ du Théorème 3.2 , ce qui termine cette preuve.

Donnons finalement le résultat suivant qui permet de construire des exemples non triviaux d'espaces localement convexes dont toutes les parties bornées sont $\sigma$-dentables.

Proposition 3.10. Soient $E$ un espace vectoriel, $\left(E_{i}, P_{i, j}\right)$ un système projectif d'espaces localement convexes (les applications $P_{i, j}$ sont linéaires et continues) indexé par $I$, et $\left(f_{i}\right)_{i \in I}$ une famille cohérente d'applications linéaires de $E$ dans $E_{i}$. On munit $E$ de la topologie localement convexe initiale associée aux applications $\left(f_{i}\right)$. Alors, pour qu'une partie $A \subset E$ soit $\sigma$-dentable (resp. linéairement $\sigma$-dentable), il faut et il suffit que pour chaque $i \in I, f_{i}(A)$ soit $\sigma$ dentable (resp. linéairement $\sigma$-dentable).

Preuve. Fixons un indice $i \in I$ et soit $V$ un voisinage de zéro dans $E_{\imath}$. Supposons la partie $A \sigma$-dentable, il existe une partie $F \subset E$ et un point $x \in A$ tels que $x \notin \sigma\left(A \backslash\left(F+f_{i}^{-1}(V)\right)\right)$. Le point $f_{i}(x)$ appartient à $f_{i}(A)$ et vérifie $f_{i}(x) \notin \sigma\left(f_{i}(A) \backslash\left(f_{i}(F)+V\right)\right)$.

Réciproquement, soit $U$ un voisinage de zéro dans $E$. On peut le supposer de la forme $U=f_{i}^{-1}(V)$ où $V$ est un voisinage de zéro dans $E_{i}$. Il existe un point $a \in f_{i}\left(A_{i}\right)$ et une partie finie $F_{i} \subset E_{i}$ tels que $\alpha \notin \sigma\left(f_{i}\left(A_{i}\right) \backslash\left(F_{i}+V\right)\right)$. Soit $x$ un point de $A_{i}$ tel que $f_{i}(x)=\alpha$; soit également $F$ une partie finie de $E$ de sorte que $f_{i}(F)=F_{i}$. Il est facile de vérifier que l'on a $x \notin \sigma\left(A_{i} \backslash(F+U)\right)$.

On démontre d'une manière identique la proposition pour les parties linéairement $\sigma$-dentables.

Remarque. On voit avec (3.10), que si pour tout $i \in I$ les bornés de $F_{i}$ sont $\sigma$-dentables, alors il en est de même pour les parties bornées de $E$. C'est par exemple le cas lorsque les espaces $E_{i}$ sont des espaces de Banach qui possèdent la propriété de Radon-Nikodym. En fait il n'est pas indispensable que la topologie $\mathscr{C}$ de $E$ soit du type de celle de la Proposition 3.10. Il suffit qu'elle soit plus fine qu'une telle topologie et que sur les parties bornées de $(E, \mathscr{C})$ les deux topologies (induites) coïncident.

La situation considérée dans la Proposition 3.10 a précisément lieu dans le cas des espaces $M_{\sigma}(T)$ étudiés par B. Ivol et J. Berruyer ainsi que dans le cas des espaces $M^{\infty}(T)$ étudiés par $M$. Rome dans [19]. Revenons sur la théorie de ces espaces en rappelant que l'on 
note par $M_{\sigma}(T)$ le dual compactologique de l'espace compactologique $\left(C^{\infty}(T), \mathscr{X}_{0}^{\infty}\right)$ où $C^{\infty}(T)$ est l'espace des fonctions continues et bornées sur un espace complètement régulier $T$ et où $\mathscr{H}_{0}^{\infty}$ désigne la compactologie constituée d'ensembles qui sont les enveloppes disquées fermées des suites équicontinues, uniformément bornées et convergentes simplement vers zéro. L'espace $M_{\sigma}(T)$ est muni de la topologie de la convergence uniforme sur les parties $H \in \mathscr{K}_{0}^{\infty}$.

En suivant [19] on désigne par $M^{\infty}(T)$ le dual compactologique de l'espace compactologique $\left(C^{\infty}(T), \mathscr{X}^{\infty}\right)$ où $\mathscr{H}^{\infty}$ désigne l'ensemble des parties équicontinues et uniformément bornées sur $T$. L'espace $M^{\infty}(T)$ est muni de la topologie de la convergence uniforme sur les parties $H \in \mathscr{H}^{\infty}$. L'intérêt de ces espaces, en ce qui nous concerne, réside dans le résultat suivant dû à Madame Bucchioni.

Proposition 3.11. Les espaces $M_{\sigma}(T)$ et $M^{\infty}(T)$ sont des elc complets dont toutes les parties bornées sont $\sigma$-dentables.

Preuve. Donnons la démonstration pour l'espace $M^{\infty}(T)$; elle se fait l'une manière identique pour les espaces $M_{\sigma}(T)$. Pour toute partition continue de l'unité $\phi=\left(\varphi_{i}\right)_{i \in I}$ on considère l'application $f_{\phi}: M^{\infty}(T) \rightarrow l^{1}(I)$ définie par $f_{\phi}(\mu)=\left(\mu\left(\phi_{i}\right)\right) i \in I$. Munissons l'espace vectoriel $M^{\infty}(T)$ de la topologie initiale $\mathscr{C} \Phi$ associée aux applications $\left(f_{\phi}\right)$ ( $\phi$ décrivant l'ensemble $\Phi$ de toutes les partitions continues de l'unité). La topologie $\mathscr{C} \Phi$ est moins fine que celle de l'espace $M^{\circ}(T)$ et, en vertu de [19], ces deux topologies admettent les mêmes parties bornées avec les mêmes topologies induites sur les parties bornées. Comme tout espace du type $l^{1}(I)$ possède la propriété de Radon-Nikodym, il résulte de la Proposition 3.10 ainsi que de la remarque ci-dessus que toute partie bornée de $M^{\infty}(T)$ est $\sigma$-dentable.

Exemple et contre-exemples. Les Théorèmes 2.4 et 2.5 donnent des conditions suffisantes pour qu'il existe une densité faible $\vec{f}$. On peut se demander si, avec ces mêmes conditions, il n'est pas possible d'obtenir mieux, par exemple, lorsque $T$ est un espace topologique, que $\vec{f}$ soit Lusin-mesurable. Il n'en est rien avec l'exemple suivant.

Exemple 1. On pose $T=[0,1], E=\boldsymbol{R}^{T}$, on prend pour $\mu$ la mesure de Lebesgue sur $T$ et on définit une mesure vectorielle $\vec{m}$ sur la tribu des ensembles $\mu$-mesurables par $\vec{m}(A)=\left(x_{t}=\int_{0}^{t} 1_{A} d \mu\right)_{t \in T}$, alors on a:

(a) $E$ est un espace de Radon-Nikodym faible et toute mesure $\mu$ provenant d'un couple $(\vec{m}, \mu)$ est de Radon.

(b) La fonction $\vec{f}$ définie par $\vec{f}(x)=\left(x_{t}\right) x_{t}=1$ si $x \leqq t$ et $x_{t}=0$ sinon, est une densité faible pour $(\vec{m}, \mu)$. 
(c) Le couple $(\vec{m}, \mu)$ n'admet pas de densité Lusin-mesurable.

Preuve. Tout est évident sauf peut-être le point (c). Pour le montrer, raisonnons par l'absurde en supposant qu'il existe une densité $\overrightarrow{f^{\prime}}$ Lusin-mesurable. Il existe donc un compact $K \subset T$ de mesure non nulle tel que la restriction de $\vec{f}^{\prime}$ à $K$ soit continue. De plus il est facile de montrer qu'il existe un point $t_{0} \in T$ tel que l'on ait à la fois $\mu\left(\left[0, t_{0}\right] \cap K\right)>0$ et $\mu\left(\left[t_{0}, 1\right] \cap K\right)>0$. Posons $B=\left[0, t_{0}\right] \cap K$ et $\overrightarrow{f_{t_{0}}^{\prime}}(x)=x_{t_{0}}$; on a évidemment $\overrightarrow{f_{t_{0}}^{\prime}}=1_{B} \mu . p . p$. dans $K$, ce qui est absurde puisque ${\overrightarrow{f_{t}}}_{t_{0}}$ est continue.

Les conditions du Théorème 3.1 sont réalisées lorsqu'il existe une densité $\vec{f}: T \rightarrow E$ Lusin-mesurable (avec $T$ espace topologique). Par contre il peut très bien exister une densité faible sans que les conditions du Théorème 3.1 aient lieu et ceci même lorsque $E$ est un espace de Banach. On peut le voir avec l'exemple suivant qui nous a été suggéré par l'étude de l'exemple que $\mathrm{E}$. Thomas considère dans le Paragraphe 2 de [22].

Exemple 2. On pose $T=[0,1]$ et $E=L^{\infty}(0,1)$; en prenant pour $\mu$ la mesure de Lebesgue sur $T$, on définit une application $\vec{f}: T \rightarrow E$ par $\vec{f}(t)=1_{[t, 1]}$. Pour tout $x^{\prime} \in L^{1}(0,1)$ on pose $\left\langle\vec{m}(A), x^{\prime}\right\rangle=\int_{A} x^{\prime} \circ \vec{f} d t$. Alors les propriétés suivantes sont réalisées:

(a ) $\vec{m}(A)$ appartient à $L^{\infty}$ pour tout $A$ appartenant à la tribu borélienne de $[0,1]$.

(b) L'application $A \rightarrow \vec{m}(A)$ est une mesure vectorielle à valeurs dans $L^{\infty}([0,1])$ (muni de la norme).

(c) La fonction $\vec{f}$ est une densité faible pour la dualité $\sigma\left(L^{\infty},\left(L^{\infty}\right)^{\prime}\right)$.

(d) La mesure $\lambda$ est de Radon sur $\left(L^{\infty}, \sigma\left(L^{\infty}, L^{1}\right)\right)$ mais elle n'est pas de Radon sur $L^{\infty}$.

(e) Aucune des conditions des Théorèmes 3.1, 3.2, et 3.3 n'est satisfaite.

(f) La fonction $\vec{f}$ n'est pas une densité Lusin-mesurable.

Preuve. Avec le théorème de Fubini on montre que $x^{\prime} \circ \vec{f}$ est $\mu$-intégrable pour tout $x^{\prime} \in L^{1}$. Donc $\vec{m}$ est une mesure à valeurs dans $\left(L^{\infty}, \sigma\left(L^{\infty}, L^{1}\right)\right)$. En fait pour tout $x^{\prime} \in\left(L^{\infty}\right)^{\prime}$ la fonction $x^{\prime} \circ \vec{f}$ est Riemann-intégrable sur $[0,1]$ donc Lebesgue-intégrable, ce qui montre encore que $\vec{m}$ est une mesure à valeurs dans $L^{\infty}$. On a ainsi prouvé les points (a), (b) et (c); pour plus de détails, on consultera [22].

Il est clair que $\lambda$ est de Radon sur $\left(L^{\infty}, \sigma\left(L^{\infty}, L^{1}\right)\right)$ puisque $\bar{S}_{T}$ est borné donc compact pour la topologie $\sigma\left(L^{\infty}, L^{1}\right)$. Remarquons 
maintenant que $\vec{m}(A)$ est la fonction de $L^{\infty}$ définie par $\vec{m}(A)(u)=\int_{0}^{u} 1_{A} d t$ qui est évidemment continue. Ainsi $\vec{m}$ est à valeurs dans l'espace $C^{\infty}([0,1])$ des fonctions continues sur $T$ alors que la densité $\vec{f}$ est à valeurs dans $L^{\infty} \mid C^{\infty}$. Supposons que $\lambda$ soit de Radon sur $L^{\infty}$; elle est portée par $C^{\infty}$ et il existe donc une densité faible $\vec{f}_{1}$ à valeurs dans $C^{\infty}$; c'est encore une densité dans l'espace souslinien $\left(L^{\infty}, \sigma\left(L^{\infty}, L^{1}\right)\right)$, on a donc $\overrightarrow{f_{1}} \neq \vec{f} \mu . p . p$. ce qui est absurde. Ainsi $\lambda$ n'est pas de Radon sur $L^{\infty}$. Comme $L^{\infty}$ est un $B-F$-espace et un "bon espace," aucune des conditions mentionnées n'est vérifiée.

De même $\vec{f}$ n'est pas Lusin-mesurable puisque $\lambda$ n'est pas de Radon.

Remarque. Pour voir que $\lambda$ n'est pas de Radon sur $L^{\infty}$ on aurait pu procéder différemment. En effet, il est clair que $\lambda$ est de Radon $\operatorname{sur}\left(L^{\infty}, \sigma\left(L^{\infty}, L^{1}\right)\right)$, que $\lambda$ est l'image par $\vec{f}$ de la mesure $\mu$ et qu'elle est portée par l'ensemble $\vec{f}(T)=\left\{1_{[t, 1]} ; t \in[0,1]\right\}$. L'ensemble $\vec{f}(T)$ est une partie discrète dans $L^{\infty}$ (pour la topologie de la norme); la mesure $\lambda$ n'étant pas atomique, elle ne peut être de Radon pour la topologie de $L^{\infty}$. On peut se demander si un elc complet $E$ dans lequel tout borné est $\sigma$-dentable est un espace de Radon-Nikodym faible (ce qui est évidemment le cas lorsque $E$ est un espace de Banach ou, plus généralement, un "bon espace"). Il n'en est rien; nous en donnerons des exemples dans le paragraphe suivant.

IV. Espaces de mesures sur un espace topologique completement regulier et propriété de Radon-Nikodym. Dans ce paragraphe, nous traitons de mesures vectorielles à valeurs dans l'espace $M^{+}(\beta T)$ des mesures de Radon positives sur le compactifié de Stone-Čech $\beta T$ de $T$. Etant donné un couple $(\vec{m}, \mu)$ avec $\vec{m}$ à valeurs dans le cône positif $M_{t}^{+}(T)$ [1] des mesures de Radon sur $T$ (resp. $M_{\sigma}^{+}(T)$ [4] des mesures de Baire positives sur $T$ ) muni de la topologie induite par la topologie étroite $\sigma\left(M(\beta T), C^{\infty}(T)\right.$ ) (de la convergence simple sur l'espace $C^{\infty}(T)$ des fonctions continues et bornées sur $T$ ) on se propose de répondre essentiellement aux questions suivantes:

(a) Existe-t-il une densité faible $\vec{f}: \Omega \rightarrow M_{t}(T)$ (resp. $\vec{f}$ : $\left.\Omega \rightarrow M_{\sigma}(T)\right)$ associée à $(\vec{m}, \mu)$ ?

(b) La mesure cylindrique $\lambda$ associée au couple $(\vec{m}, \mu)$ est-elle de Radon sur $M_{t}(T)$ (resp. $\left.M_{\sigma}(T)\right)$ ?

Dans le cas de l'espace $M_{t}(T)$ la réponse est, comme nous allons l'établir, affirmative. On a même mieux car, non seulement $\lambda$ est de Radon, mais en plus, pour tout $\varepsilon>0$, elle est à $\varepsilon$-près concentrée sur des parties de $T$ vérifiant la condition de Prokhorov [1], ce qui 
est tout à fait exceptionnel puisque hors mis le cas où $T$ est un espace de Prokhorov (par exemple polonais ou localement compact) les compacts de $M_{t}^{+}(T)$ ne satisfont pas la condition de Prokhorov.

Par contre, dans le cas de l'espace $M_{\sigma}(T)$, il n'existe géneralement pas de densité faible $\vec{f}$ associée au couple $(\vec{m}, \mu)$, ce qui implique d'ailleurs que $\lambda$ n'est pas de Radon. Dans le cas particulier où $T$ est métrisable separable, le fait que tout couple $(\vec{m}, \mu)$ soit densitable équivaut à la condition: $T$ est radonien [1] et on a alors $M_{t}(T)=M_{\sigma}(T)$.

Ce dernier résultat va nous fournir, comme nous l'avons signalé à la fin du Paragraphe III, des exemples d'espaces qui sont complets, dont tous les bornés sont $\sigma$-dentables et qui ne sont pas pour autant des espaces de Radon-Nikodym faibles.

Pour toute partie $H \subset M_{t}(T)$ on notera par $\bar{\Gamma}(H)$ son enveloppe disquée fermée et par $\bar{\Gamma}_{+}(H)$ la trace de $\bar{\Gamma}(H)$ sur le cône des mesures positives. Rappelons que l'ensemble $S_{\Omega}=(\vec{m}(A) / \mu(A) ; A \epsilon$ $\Sigma, \mu(A) \neq 0)$ est supposé borné dans $M_{t}(T)$.

Commençons par traiter le cas particulier où $T$ est un espace compact.

THÉORÈmE 4.1. Supposons que T est compact et soit $(\vec{m}, \mu)$ avec $\vec{m}: \Sigma \rightarrow M^{+}(T)$ une mesure vectorielle à valeurs dans le cône positif $M^{+}(T)$ des mesures de Radon sur $T$ muni de la topologie étroite $\sigma\left(M(T), C^{\infty}(T)\right)$. Alors les conditions suivantes sont réalisées:

(a) Il existe une densité faible $\vec{f}: \Omega \rightarrow M^{+}(T)$ associée au couple $(\vec{m}, \mu)$ telle que $\vec{f}(\Omega) \subset \bar{\Gamma}_{+}\left(S_{\Omega}\right)$.

(b) La mesure cylindrique $\lambda$ associée au couple $(\vec{m}, \mu)$ est de Radon $\operatorname{sur} M^{+}(T)$.

Preuve. Par hypothèse l'ensemble $S_{\Omega}$ est borné dans $M(T)$, donc relativement compact (pour la topologie étroite); il résulte donc de (2.1) que $\lambda$ est de Radon et portée par l'adhérence $\bar{S}_{\Omega}$ de $S_{\Omega}$ dans $M(T)$. Comme $\bar{S}_{\Omega}$ est lui-même inclus dans le cône positif $M^{+}(T)$, le point (b) est acquis.

Pour établir (a) on procède comme dans la preuve ce (1.3). $\mathrm{Si}$ $\rho$ est un relèvement $\operatorname{sur} L^{\infty}(\Omega, \Sigma, \mu)$, pour toute point $\omega \in \Omega$ et pour toute fonction $g \in C^{\infty}(T)$ on pose $\langle\vec{f}(\omega), g\rangle=\rho\left(f_{g}\right)(\omega)$ où $f_{g}$ désigne la densité scalaire (en fait sa classe dans $L^{\infty}(\Omega, \Sigma, \mu)$ ) associée au couple $(g \circ \vec{m}, \mu)$. On vérifie facilement que pour toute $g$ appartenant au polaire $S_{\Omega}^{0}$ on a $N^{\infty}\left(f_{g}\right) \leqq 1$ donc $\left|\rho\left(f_{g}\right)(\omega)\right| \leqq 1$ pour tout $\omega \in \Omega$. Ainsi la fonction $\vec{f}: \omega \rightarrow \vec{f}(\omega)$ est une densité associée au couple $(\vec{m}, \mu)$ et on a $\vec{f}(\Omega) \subset \bar{\Gamma}\left(S_{\Omega}\right)$.

De plus, pour tout $A \in \Sigma$ et pour toute fonction positive $g \in C_{+}^{\infty}(T)$ 
on a $\langle\bar{m}(A), g\rangle=\int_{A} f_{g} d \mu \geqq 0$; il en résulte que $f_{g} \geqq 0 \mu$-presque partout et, en vertu d'une des propriétés du relèvement [10], on a $\rho\left(f_{g}\right) \geqq 0$ partout. Ainsi pour toute $g \in C_{+}^{\infty}(T)$ on a $\langle\vec{f}(\omega), g\rangle \geqq 0$, ce qui signifie précisément que $\vec{f}$ est à valeurs dans le cône positif $M^{+}(T)$.

REMARque. On aurait pu, soit en utilisant la technique des dérivants de J. Pellaumail [17] et J. Kupka [12] soit à l'aide du Théorème 2.6, affiner quelque peu le point (a) et établir qu'il existe une densité à valeurs dans l'adhérence $\bar{S}_{\Omega}$.

Passons maintenant au cas où $T$ est complètement régulier quelconque.

THÉoRÈme 4.2. Soit $T$ un espace complètement régulier. Considérons un couple $(\vec{m}, \mu)$ avec $\vec{m}: \Sigma \rightarrow M_{t}^{+}(T)$ une mesure à valeurs dans le cône positif des mesures de Radon sur $T$. On a alors

(a) Il existe une densité faible $\vec{f}: \Omega \rightarrow M_{t}^{+}(T)$ associée au couple $(\vec{m}, \mu)$ telle que $\vec{f}(\Omega)$ soit borné dans $M_{t}^{+}(T)$.

(b) La mesure cylindrique $\lambda$ associée au couple $(\vec{m}, \mu)$ est de Radon sur $M_{t}^{+}(T)$. De plus, pour tout $\varepsilon>0$, il existe une partie $H_{\varepsilon} \subset M_{t}^{+}(T)$ satisfaisant à la condition de Prokhorov et telle que $\lambda$ soit concentrée à $\varepsilon$-près sur $H_{\varepsilon}$.

Preuve.

(a) Commençons par remarquer que pour tout $A \in \Sigma$ la mesure $\vec{m}(A)$ est absolument continue par rapport à la mesure $\vec{m}(\Omega)$. Soit maintenant $\left(K_{n}\right), n \geqq 1$, une suite de parties compactes de $T$ deux à deux disjointes et telle que l'ensemble $T \backslash\left(\bigcup_{1}^{+\infty} K_{n}\right)$ soit $\vec{m}(\Omega)$-négligeable (donc encore $\vec{m}(A)$-négligeable pour tout $A \in \Sigma$ ). Pour tout $n \geqq 1$, définissons une application $\vec{m}_{n}: \Sigma \rightarrow M^{+}\left(K_{n}\right)$ par $\vec{m}_{n}(A)=1_{K_{n}}$. $\vec{m}(A)$ où $1_{K_{n}}$ désigne évidemment la fonction indicatrice du compact $K_{n}$. On a le résultat élémentaire suivant

LEMME 1. L'application $\vec{m}_{n}$ est une mesure vectorielle.

Preuve du lemme. Soit $\left(A_{p}\right), p \geqq 1$, une suite d'éléments de $\Sigma$ deux à deux disjoints. Alors pour tout compact $L \subset T$ on a

$$
\sum_{p=1}^{+\infty} \vec{m}_{n}\left(A_{p}\right)(L)=\sum_{p=1}^{+\infty} \vec{m}\left(A_{p}\right)\left(K_{n} \cap L\right)=\vec{m}\left(\bigcup_{1}^{+\infty} A_{p}\right)\left(K_{n} \cap L\right)=\vec{m}_{n}\left(\bigcup_{1}^{+\infty} A_{n}\right)(L)
$$

ce qui termine la preuve du lemme.

D'après le Théorème 4.1 il existe une densité faible $\vec{f}_{n}: \Omega \rightarrow M^{+}\left(K_{n}\right)$ associée au couple $\left(\vec{m}_{n}, \mu\right)$. L'application $\omega \rightarrow \sum_{n=1}^{+\infty}\left\langle\vec{f}_{n}(\omega), 1_{T}\right\rangle$ de $\Omega$ dans $\bar{R}^{+}$est évidemment $\mu$-mesurable, donc pour tout $A \in \Sigma$ on a 


$$
\begin{aligned}
\int_{\Delta} \sum_{n=1}^{+\infty}\left\langle\vec{f}_{n}(\omega), 1_{T}\right\rangle d \mu(\omega) & =\sum_{n=1}^{+\infty} \int_{A}\left\langle f_{n}(\omega), 1_{T}\right\rangle d \mu(\omega) \\
& =\sum_{n=1}^{+\infty} \vec{m}_{n}(A)\left(K_{n}\right)=\vec{m}(A)(T)<+\infty .
\end{aligned}
$$

Il en résulte que $\sum_{n=1}^{+\infty} f_{n}(\omega) \in M_{t}^{+}(T) \mu . p . p$.

Ainsi en posant $\vec{f}(\omega)=\sum_{n=1}^{+\infty} \vec{f}_{n}(\omega)$ aux points $\omega$ où la suite $\left(\overrightarrow{f_{n}}(\omega)\right)$ est sommable dans $M_{t}^{+}(T)$, et $\overrightarrow{f(}(\omega)=\overrightarrow{0}$ ailleurs, on obtient une densité associée au couple $(\vec{m}, \mu)$. De plus il est tout à fait évident que l'on a

$$
\sum_{1}^{+\infty}\left\langle\vec{f}_{n}(\omega), 1_{T}\right\rangle \leqq \sup _{\nu \in S_{2}}(\nu(T))<+\infty
$$

$\mu$-presque partout, donc la densité $\vec{f}$ peut être prise à valeurs dans une partie bornée de $M_{t}^{+}(T)$. Ce qui termine la preuve du point (a).

(b) Nous venons de voir ci-dessus que la série $\sum_{1}^{+\infty}\left\langle\vec{f}_{n}(\omega), 1_{T}\right\rangle$ converge $\mu$-presque partout. Il résulte du théorème d'Egoroff qu'elle converge $\mu$-presque uniformément. Fixons $\varepsilon>0$; il existe donc $A \in \Sigma, \mu(T \backslash A) \leqq \varepsilon$, de sorte que la série $\sum_{1}^{+\infty}\left\langle\vec{f}_{n}(\omega), 1_{T}\right\rangle$ converge uniformément sur $A$. Posons $L_{\varepsilon}=\vec{f}(A)=\left\{\nu=\sum_{1}^{+\infty} \vec{f}_{n}(\omega) ; \omega \in A\right\}$.

On a alors le résultat suivant

LEMME 2. La partie $L_{\varepsilon}$ satisfait a la condition de Prokhorov.

Preuve $d u$ lemme. Fixons $\varepsilon^{\prime}>0$; il existe un entier $N>0$ tel que l'on ait $\sum_{N+1}^{+\infty}\left\langle\overrightarrow{f_{n}}(\omega), 1_{T}\right\rangle \leqq \varepsilon^{\prime}$ pour tout $\omega \in A$. Mais cela signifie exactement que pour tout $\nu \in L_{\varepsilon}$ on a $\nu\left(T \backslash\left(\bigcup_{1}^{N} K_{n}\right)\right) \leqq \varepsilon^{\prime}$ ce qui, joint au fait que $\vec{f}(\Omega)$ est borné dans $M_{t}^{+}(T)$, prouve le lemme.

Pour terminer nous avons besoin du résultat élémentaire suivant

Lemme 3. Soit $A \in \Sigma, \mu(A) \neq 0$, alors l'ensmble $S_{A}$ est inclus dans l'enveloppe disquée fermée $\bar{\Gamma}(\vec{f}(A))$ de l'ensemble $\vec{f}(A)$.

Preuve du lemme. Cest une conséquence immédiate du théorème de Hahn-Banach. En effet, si $g$ appartient au polaire $\vec{f}(A)^{0}$, alors pour tout $B \in \sum_{A}, \mu(B) \neq 0$

$$
\left|\frac{1}{\mu(B)} \int_{B}\langle\vec{f}(t), g\rangle c^{\prime} \mu\right|=\left|\left\langle\frac{\vec{m}(B)}{\mu(B)}, g\right\rangle\right| \leqq 1
$$

ce qui suffit.

Revenons à la démonstration du point (b). L'enveloppe disquée fermée d'une partie de $M_{t}(T)$ vérifiant la condition de Prokhorov la vérifie également. Il résulte des Lemmes 1 et 2 que pour tout $\varepsilon>0$ il existe $A \in \Sigma, \mu(T \backslash A) \leqq \varepsilon$ et une partie de Prokhorov $H_{\varepsilon}=\bar{\Gamma}_{+}\left(L_{\varepsilon}\right)$ 
de sorte que $S_{A} \subset H_{\varepsilon}$. D'après le Théorème 2.1, la mesure cylindrique $\lambda$ est cylindriquement concentrée à $\varepsilon$-près sur $H_{\varepsilon}$ mais les ensembles de Prokhorov étant relativement compacts dans $M_{t}(T)$ la mesure cylindrique $\lambda$ est de Radon sur $M_{t}^{+}(T)$.

Nous allons maintenant considerer le cas de l'espace $M_{\sigma}^{+}(T)$ des mesures de Baire sur $T$. Comme nous l'avons dit au Paragraphe III, on peut soit munir $M_{\sigma}(T)$ de la topologie de la $\mathscr{H}_{0}^{\infty}$-convergence [4] soit de la topologie étroite $\sigma\left(M_{\sigma}(T), C^{\infty}(T)\right)$. En fait, pour des problèmes du type Radon-Nikodym, il est indifférent de considérer l'une ou l'autre topologie. En effet, ces deux topologies admettent les mêmes parties compactes et les mêmes parties bornées. Les mesures vectorielles à valeurs dans $M_{\sigma}(T)$ sont donc les mêmes pour ces deux topologies, les couples $(\vec{m}, \mu)$ également. Il est clair aussi qu'elles admettent les mêmes mesures cylindriques et les mêmes mesures de Radon.

Rappelons maintenant les définitions suivantes:

DEFINITION 4.4. Un espace topologique $T$ est dit radonien [1] lorsque toute mesure borélienne positive et bornée sur $T$ est de Radon.

DeFINITION 4.5. Un espace topologique $T$ est dit universellement Radon-mesurable [21] lorsqu'il est une partie universellement Radon-mesurable du compactifié de Stone-Čech $\beta T$ de $T$.

Rappelons également que l'on a $M_{\sigma}(T)=M_{\sigma}(\nu T)$ où $\nu T$ désigne le replété de $T$ [4]. Ainsi, pour alléger l'écriture, on suppose dans toute la suite que $T$ est replet c'est-à-dire $T=\nu T$.

On peut déjà donner pour un espace complètement régulier $T$ quelconque le résultat suivant

THÉORÈme 4.6. Supposons que pour tout couple $(\vec{m}, \mu)$, avec $\vec{m}: \Sigma \rightarrow M_{\sigma}^{+}(T)$ à valeurs dans le cône positif $M_{\sigma}^{+}(T)$, la mesure cylindrique associée $\lambda$ soit de Radon. Alors l'espace $T$ est universellement Radon-mesurable.

Preuve. Commençons par établir le lemme préparatoire suivant

LEMme 1. Supposons que T ne soit pas universellement Radonmesurable. Il existe alors une probabilité de Radon $\mu$ sur $\beta T$ telle que $T$ soit de mesure intérieure $\mu_{*}(T)$ nulle et de mesure extérieure $\mu^{*}(T)$ égale à un.

Preuve du lemme. Il est immédiat que si $T$ n'est pas Radon 
universellement mesurable alors il existe une mesure de Radon $\eta$ sur $\beta T$ telle que l'on ait $\eta_{*}(T)=\alpha<\eta^{*}(T)=\beta$. Soit $\left(K_{n}\right)$ une suite de parties compactes de $T$ telle que $\alpha=\eta\left(\cup K_{n}\right)$, alors la mesure $\nu=1_{T \backslash \cup K_{n}} \cdot \eta$ est telle que $\nu_{*}(T)=0$ et $\nu^{*}(T)=\beta-\alpha$. Soit finalement un ouvert $L$ de $\beta T$ contenant $T$ et tel que $\nu(L)=\beta-\alpha$, alors la mesure $1 /(\beta-\alpha) \cdot 1_{L} \cdot \nu=\mu$ satisfait aux conditions désirées.

Revenons à la preuve du théorème. Notons par $\vec{m}$ la mesure vectorielle sur la tribu borélienne $\mathscr{B}(\beta T)$, à valeurs dans $M_{\sigma}^{+}(T)$, définie par $\langle\vec{m}(A), B\rangle=\mu^{*}(A \cap B)$ pour tout borélien $A \in \mathscr{B}(\beta T)$ et toute partie de Baire $B \in \mathscr{B}_{a}(T)$. En vertu des hypothèses, la mesure cylindrique $\lambda$ associée à $(\vec{m}, \mu)$ est de Radon. Fixons $\varepsilon>0$, il existe donc un compact $K \subset \beta T, \mu(\beta T \backslash K) \leqq \varepsilon$ et un disque compact $D_{\varepsilon} \subset M_{\sigma}(T)$ tels que $S_{K} \subset D_{\varepsilon}$. On peut même supposer que le compact $K$ est le support de la mesure induite $\mu_{K}$ sur $K$. Il est également clair que $K$ n'est pas inclus dans $T$. Soit donc $a \in K \backslash T$; par hypothèse $T=\nu T$; ainsi il existe une fonction continue $f$ sur $\beta T$ qui vaut zéro au point a et qui ne s'annule pas sur $T$. Pour tout entier $n \geqq 1$, posons $F_{n}=\{x \in K ;|f(x)| \leqq(1 / n)\}$ et $H_{n}=F_{n} \cap T \in \mathscr{B}_{a}(T)$. Alors d'une part l'intérieur $\dot{F}_{n}$ est non vide donc $\mu\left(F_{n}\right) \neq 0$ et d'autre part $\cap H_{n}=\varnothing$.

La suite $\left(\nu_{n}=\left(\vec{m}\left(F_{n}\right) / \mu\left(F_{n}\right)\right)\right)$ est incluse dans $D_{\varepsilon}$ donc relativement compacte. D'après [1] on doit avoir $\sup _{n \geq 1}\left|\nu_{n}\left(H_{m}\right)\right| \rightarrow_{m \rightarrow+\infty} 0$, ce qui est évidemment absurde puisque $\nu_{n}\left(H_{n}\right)=1$.

On s'aperçoit donc déjà du rôle important que vont tenir les propriétés de mesurabilité de $T$ pour que tout couple $(\vec{m}, \mu)$ $\left(\vec{m}: \Sigma \rightarrow M_{\sigma}^{+}(T)\right)$ soit densitable. Dans le cas particulier où $T$ est métrisable séparable on a la caractérisation très précise suivante.

THÉorème 4.7. Soit $T$ un espace métrisable séparable. Les conditions suivantes sont équivalentes.

(a) $T$ est radonien

(b) $M_{\sigma}(T)=M_{t}(T)$

(c) T est Radon-universellement mesurable.

(d) Pour tout couple $(\vec{m}, \mu)$ avec $\vec{m}: \Sigma \rightarrow M_{\sigma}^{+}(T)$ la mesure cylindrique associée $\lambda$ est de Radon.

(e) Pour tout couple $(\vec{m}, \mu)$ avec $\vec{m}: \Sigma \rightarrow M_{\sigma}^{+}(T)$ il existe une densité faible associée $\vec{f}: \Omega \rightarrow M_{\sigma}(T)$.

Preuve. (a) $\Leftrightarrow(\mathrm{b})$ : c'est immédiat compte tenu de la définition des espaces radoniens puisque sur $T$ les mesures de Borel et les mesures de Baire sont exactement les mêmes.

$(a) \Leftrightarrow(c)$ : c'est immédiat également d'après [1] puisque le completé de $T$ est polonais donc radonien.

(b) $\Rightarrow(d)$ : c'est le Théorème 4.2 . 
$(\mathrm{d}) \Rightarrow(\mathrm{e})$ : ce point résulte du Théorème 2.4 .

$(\mathrm{e}) \Rightarrow(\mathrm{c})$ : c'est le point difficile. Supposons que $T$ n'est pas universellement Radon-mesurable, alors en vertu du lemme du Théorème 4.6 il existe une probabilité $\mu \in M(\beta T)$ telle que l'on ait $\mu_{*}(T)=0$ et $\mu^{*}(T)=1$. On définit une mesure borélienne $\mu_{T}$ sur $T$ par $\mu_{T}(B)=\mu\left(B^{\prime}\right)$ avec $B^{\prime}$ borélien quelconque de $T$ vérifiant $B^{\prime} \cap T=B$. On considère maintenant l'application mesurable $h:(T, \mathscr{B}(T)) \rightarrow(T \times \beta T, \mathscr{B}(T) \otimes \mathscr{B}(\beta T))$ définie par $h(t)=(t, t)$ et notons par $\nu$ la mesure image par l'application $h$ de la mesure $\mu_{T}$. Tout repose sur le lemme suivant

Lemme 1. Il n'existe pas de désintégration stricte $\left(\nu_{s}\right), s \in \beta T$, de la mesure $\nu$.

Preuve du lemme. Supposons qu'il existe une désintégration $\left(\nu_{s}\right), s \in \beta T$, de $\nu$. Pour tout ensemble $A \in \mathscr{B}(T) \otimes \mathscr{B}(\beta T)$ l'application $s \rightarrow \nu(A(\cdot, s))$ est $\mu$-mesurable et l'on a $\nu(A)=\int \nu_{s}(A(\cdot, s)) d \mu(s)$. En effet, on remarquera que la mesure image de $\nu$ par la projection sur $T$ c'est précisément la mesure $\mu$. La diagonale $\Delta=\{(t, t) ; t \in T\}$ appartient à la tribu produit $\mathscr{B}(T) \otimes \mathscr{B}(\beta T)$ (qui, $T$ étant métrisable séparable, coïncide avec la tribu borélienne $\mathscr{B}(T \times \beta T)$ de l'espace produit) et l'on a $\nu(\Delta)=1$, mais comme l'ensemble $B=\left\{s ; \nu_{s}(s) \neq 0\right\}$ est $\mu$-négligeable on a également $\int \nu_{s}(\Delta(\cdot, s)) d \mu(s)=0$. On obtient donc une contradiction.

Revenons à la preuve de $(\mathrm{e}) \Rightarrow(\mathrm{c})$. Considérons la mesure vectorielle $\vec{m}: \mathscr{B}(\beta T) \rightarrow M_{\sigma}^{+}(T)$ définie par $\vec{m}(A)(B)=\nu(A \times B)=$ $\mu^{*}(A \cap B)$ pour tout $A \in \mathscr{B}(\beta T)$ et toute partie de Baire $B \in \mathscr{B}_{a}(T)$. Par hypothèse il existe une densité faible $f: \beta T \rightarrow M_{\sigma}(T)$ associée au couple $(\vec{m}, \mu)$. On vérifie facilement que la famille $(\vec{f}(s)), s \in \beta T$, est une désintégration de $\nu$, ce qui est absurde en vertu du Lemme 1.

REMARQUE. Lorsque $T$ est métrisable séparable on a évidemment $M_{\tau}(T)=M^{\infty}(T)=M_{\sigma}(T)$ (voir [4] et [19]).

On tire du Théorème 4.6 l'exemple suivant (annoncé à la fin du Paragraphe III).

ExEmPle 4.8. Soit $\mu$ la mesure de Lebesgue sur $I=[0,1]$ et soit $\Omega \subset[0,1]$ un ensemble non $\mu$-mesurable. On munit l'espace $M_{\sigma}(\Omega)=M^{\infty}(\Omega)$ de la topologie de la $\mathscr{H}_{0}^{\infty}$-convergence [4]. Alors les propriétés suivantes sont réalisées.

(a) $M_{\sigma}(\Omega)$ est un elc complet et toute partie bornée $B \subset M_{\sigma}(\Omega)$ est $\sigma$-dentable.

(b) L'espace $M_{\sigma}(\Omega)$ n'est pas un espace de Radon-Nikodym faible. 
Preuve. La condition (a) est satisfaite pour tout espace $M_{o}(T)$. La condition (b) résulte de (4.6).

Remarque. Dans la démonstration de l'implication $(\mathrm{e}) \Rightarrow(\mathrm{c})$, le fait que $T$ soit métrisable séparable permet d'assurer l'égalité des tribus $\mathscr{B}(T) \otimes \mathscr{B}(\beta T)=\mathscr{B}(T \times \beta T)$. $\quad \mathrm{Si}$ on suppose que $T$ est seulement métrisable, on n'a plus cette égalité. Tout ce que nous sommes en mesure de dire se résume dans la proposition suivante.

Proposition 4.9. Soit $(T, d)$ un espace métrique qui n'est pas universellement Radon-mesurable. Alors il existe un sous-espace fermé séparable $\Omega \subset T$ tel que $M_{\sigma}(\Omega)$ ne soit pas un espace de RadonNikodym faible.

Preuve. Reprenons la preuve de (4.6). Soit $\mu$ la probabilité sur $\beta T$ telle que $\mu_{*}(T)=0$ et $\mu^{*}(T)=1$. Tout résulte du lemme suivant.

Lemme. Soit $S$ le support de la mesure $\mu$ alors l'ensemble $\Omega=S \cap T$ est métrisable séparable.

Preuve du lemme. Sur $\Omega=S \cap T$ considérons la mesure $\mu_{\omega}$ définie par $\mu_{\omega}(B)=\mu\left(B^{\prime}\right)$ avec $B \in \mathscr{B}(\Omega), B^{\prime} \in \mathscr{B}(S)$ et $B^{\prime} \cap \Omega=B$. Il est clair que la mesure $\mu_{\omega}$ est bien définie et qu'elle admet comme support l'espace tout entier. Fixons $n \geqq 1$ et soit $M_{n}$ un ensemble maximal (pour la relation d'inclusion) dans la famille des parties $M$ de $\Omega$ vérifiant: $x, y \in M \quad x \neq y \Rightarrow d(x, y)>1 / n$. Il est clair que l'ensemble $M_{n}$ est dénombrable et que la réunion $M=\bigcup_{n \geqq 1} M_{n}$ est dense dans $\Omega$.

Ainsi, partant d'un ensemble métrisable $T$ non universellement Radon-mesurable, on se ramène à un ensemble $\Omega$ avec ces deux mêmes propriétés qui, de plus, est séparable et fermé dans $T$. Une démonstration point à point identique à celle du Théorème 4.6 permet de voir que l'espace $M_{\sigma}(\Omega)$ n'est pas un espace de RadonNikodym faible.

\section{BIBLIOGRAPHIE}

1. A. Badrikian, Séminaire sur les fonctions aléatoires linéaires et les mesures cylindriques, Lecture Notes $\mathrm{n}^{\circ} 139$ (1970).

2. A Badrikian et S. Chevet, Questions liées à la théorie des espaces de Wiener, Ann. Inst. Fourier, Grenoble, 24, 2 (1974), 1-25.

3. A. Badrikian et S. Chevet, Mesures cylindriques, espaces de Wiener et fonctions aléatoires gaussiennes, Lectures Notes $\mathrm{n}^{\circ} 379$ (1974).

4. J. Berruyer et B. Ivol, Espaces de mesures et compactologies, Publ. Dép. Math. Lyon (1972), t. 9-1, p. 1-35.

5. J. P. R. Christensen, Borel structures and a topological zero-one Law, Math. Scand., 
29 (1971), 245-255.

6. A. Goldman et D. Bucchioni, Sur certains espaces de formes linéaires liés aux mesures vectorielles, à paraître aux Ann. Inst. Fourier, XXVI, 3 (1976).

7. D. J. Hebert, Jr., A general theorem for decomposition of linear random processes, Proc. Amer. Math. Soc., 38, $\mathrm{n}^{\circ} 2$ (1973), 331-336.

8. D. J. Hebert, Jr., Vector valued measures and decomposing maps, Symposium on vector and operator valued measures and applications at snowbird resort, Alta, Utah, August 1972, 7-12.

9. R. E. Huff, Dentability and the Radon-Nikodym property, Duke Math. J., 41 (1974), 111-114.

10. A. Ionescu Tulcea and C. Ionescu Tulcea, On the lifting property (II), J. Math. Mech., 11 (1962), 773-796.

11. A. Ionescu Tulcea and C. Ionescu Tulcea, Topics in the Theory of Liftings, BerlinHeidelberg-New York, Springer, 1969.

12. J. Kupka, Radon-Nikodym theorems for vector valued measures, Trans. AMS, 169 (1972), 197-217.

13. Kwapien, On a theorem of $L$. Schwartz and its applications to absolutely summing operators, Studia Math., XXXVIII (1970), 193-201.

14. A. Liapounov, Sur les fonctions-vecteurs complètement additives, Bull. Acad. Sc. URSS, Ser. Math., 4 (1940), 465-478.

15. H. Maynard, A geometrical characterization of Banach spaces with the RadonNikodym property, Trans. Amer. Math. Soc., 185 (1973), 493-500.

16. M. Metivier, Martingales à valeurs vectorielles, Application à la dérivation des mesures vectorielles, Ann. Inst. Fourier, 2 (1967), 175-208.

17. J. Pellaumail, Sur la dérivation d'une mesure vectorielle, Bull. Soc. Math. France,

98 (1970), 359-368.

18. M. A. Rieffel, Dentable subsets of Banach spaces with applications to a RadonNikodym theorem, Proc. Conf. Funct. Analysis (Irvine Calif., 1966) Thompson Book Co., Washington, 1967, p. 71-77, MR 36 \# 5668.

19. M. Rome, Ordre et compacité dans l'espace $M^{\infty}(T)$, Comptes rendus, 247, série A (1972), 1817.

20. L. Schwartz, Propriété de Radon-Nikodym, Séminaire Maurey-Schwartz, Ecole Polytechnique, exposés $\mathrm{n}^{\circ} 4$, 5, 6, année 1974-1975.

21. C. Sunyach, Une caractérisation des espaces universellement Radon-mesurables, Comptes rendus, 268, série A (1969), 864.

22. E. Thomas, Integration of functions in locally convex Suslin spaces, Trans. Amer. Math. Soc., 212 (1975), 61-81.

23. I. Tweddle, The range of a vector-valued measure, Glasgow Math. J., 13 (1972), $64-68$.

24. J. J. Uhl, Jr., A note on the Radon-Nikodym property for Banach spaces, Revue Roum. Math. pures et appl., 17 (1) (1972), 113-115.

Received May 13, 1976

Département De Mathématiques

Université Claude-Bernard

43, BD DU ONZE NOVEMbRe 1918

69621-VILLEURBANNE

France 



\section{PACIFIC JOURNAL OF MATHEMATICS}

\section{EDITORS}

RICHARD ARENS (Managing Editor)

University of California

Los Angeles, CA 90024

R. A. BEAUMONT

University of Washington

Seattle, WA 98105

C. C. Moore

University of California

Berkeley, CA 94720

\section{J. DugundJI}

Department of Mathematics

University of Southern California

Los Angeles, CA 90007

R. Finn and J. Milgram

Stanford University

Stanford, CA 94305

\section{ASSOCIATE EDITORS}

\section{E. F. BECKENBACH}

B. H. NEUMANN

F. WoLF

K. YOSHIDA

\section{SUPPORTING INSTITUTIONS}

\author{
UNIVERSITY OF BRITISH COLUMBIA \\ CALIFORNIA INSTITUTE OF TECHNOLOGY \\ UNIVERSITY OF CALIFORNIA \\ MONTANA STATE UNIVERSITY \\ UNIVERSITY OF NEVADA \\ NEW MEXICO STATE UNIVERSITY \\ OREGON STATE UNIVERSITY \\ UNIVERSITY OF OREGON \\ OSAKA UNIVERSITY
}

\author{
UNIVERSITY OF SOUTHERN CALIFORNIA \\ STANFORD UNIVERSITY \\ UNIVERSITY OF HAWAII \\ UNIVERSITY OF TOKYO \\ UNIVERSITY OF UTAH \\ WASHINGTON STATE UNIVERSITY \\ UNIVERSITY OF WASHINGTON \\ AMERICAN MATHEMATICAL SOCIETY
}

The Supporting Institutions listed above contribute to the cost of publication of this Journal, but they are not owners or publishers and have no responsibility for its content or policies.

Mathematical papers intended for publication in the Pacific Jaurnal of Mathematics should be in typed form or offset-reproduced, (not dittoed), double spaced with large margins. Please do not use built up fractions in the text of your manuscript. You may however, use them in the displayed equations. Underline Greek letters in red, German in green, and script in blue. The first paragraph or two must be capable of being used separately as a synopsis of the entire paper. Items of the bibliography should not be cited there unless absolutely necessary, in which case they must be identified by author and Journal, rather than by item number. Manuscripts, in triplicate, may be sent to any one of the editors. Please classify according to the scheme of Math. Reviews, Index to Vol. 39. All other communications should be addressed to the managing editor, or Elaine Barth, University of California, Los Angeles, California, 90024.

The Pacific Journal of Mathematics expects the author's institution to pay page charges, and reserves the right to delay publication for nonpayment of charges in case of financial emergency

100 reprints are provided free for each article, only if page charges have been substantially paid. Additional copies may be obtained at cost in multiples of 50 .

The Pacific Journal of Mathematics is issued monthly as of January 1966. Regular subscription rate: $\$ 7200$ a year (6 Vols., 12 issues). Special rate: $\$ 36.00$ a year to individual members of supporting institutions.

Subscriptions, orders for back numbers, and changes of address should be sent to Pacific Journal of Mathematics, 103 Highland Boulevard, Berkeley, California, 94708.

PUBLISHED BY PACIFIC JOURNAL OF MATHEMATICS, A NON-PROFIT CORPORATION

Printed at Kokusai Bunken Insatsusha (International Academic Printing Co., Ltd.). 8-8, 3-chome, Takadanobaba, Shinjuku-ku, Tokyo 160, Japan.

Copyrit (C) 1975 by Pacific Journal of Mathematics Manufactured and first issued in Japan 


\section{Pacific Journal of Mathematics \\ Vol. 69, No. $2 \quad$ June, 1977}

Carol Alf and Thomas Alfonso O'Connor, Unimodality of the Lévy spectral

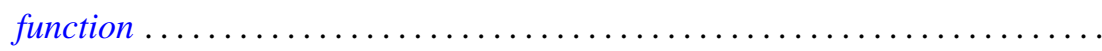

S. J. Bernau and Howard E. Lacey, Bicontractive projections and reordering of

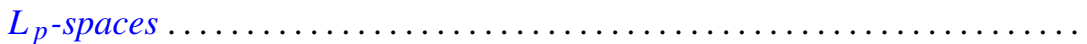

Andrew J. Berner, Products of compact spaces with bi-k and related spaces..... 303

Stephen Richard Bernfeld, The extendability and uniqueness of solutions of ordinary differential equations ...............................

Marilyn Breen, Decompositions for nonclosed planar m-convex sets ..........

Robert F. Brown, Cohomology of homomorphisms of Lie algebras and Lie

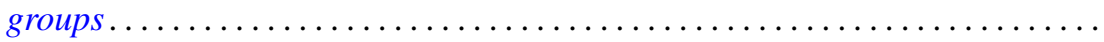

Jack Douglas Bryant and Thomas Francis McCabe, A note on Edelstein's iterative test and spaces of continuous functions ....................

Victor P. Camillo, Modules whose quotients have finite Goldie dimension ....... 333

David Downing and William A. Kirk, A generalization of Caristi's theorem with

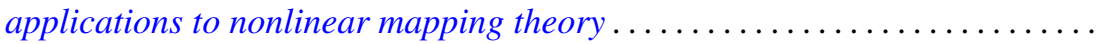

Daniel Reuven Farkas and Robert L. Snider, Noetherian fixed rings ...........

Alessandro Figà-Talamanca, Positive definite functions which vanish at

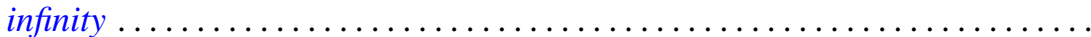

Josip Globevnik, The range of analytic extensions .................. 365

André Goldman, Mesures cylindriques, mesures vectorielles et questions de

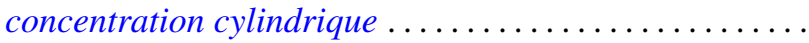

Richard Grassl, Multisectioned partitions of integers..........

Haruo Kitahara and Shinsuke Yorozu, A formula for the normal part of the

Laplace-Beltrami operator on the foliated manifold .... .

Marvin J. Kohn, Summability $R_{r}$ for double series .........

Charles Philip Lanski, Lie ideals and derivations in rings with involution ..

Solomon Leader, A topological characterization of Banach contractions . .

Daniel Francis Xavier O’Reilly, Cobordism classes of fiber bundles . .

James William Pendergrass, The Schur subgroup of the Brauer group . .

Howard Lewis Penn, Inner-outer factorization of functions whose Fourier series

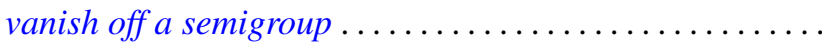

501

William T. Reid, Some results on the Floquet theory for disconjugate definite

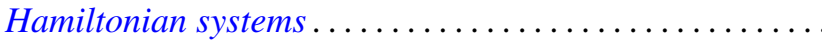

Caroll Vernon Riecke, Complementation in the lattice of convergence

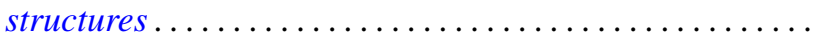

Louis Halle Rowen, Classes of rings torsion-free over their centers ......... 527

Manda Butchi Suryanarayana, A Sobolev space and a Darboux problem ....... 535

Charles Thomas Tucker, II, Riesz homomorphisms and positive linear maps.... 551

William W. Williams, Semigroups with identity on Peano continua ........... 557

Yukinobu Yajima, On spaces which have a closure-preserving cover by finite 